\title{
Structure of the Semi-Classical Amplitude for General Scattering Relations
}

\author{
Ivana Alexandrova \\ Department of Mathematics, University of Toronto, Toronto, Ontario, Canada M5S 3G3, \\ Tel.: 1-416-946-0318, Fax: 1-416-978-4107, email: alexandr@math.toronto.edu \\ July 26, 2004
}

\begin{abstract}
We consider scattering by general compactly supported semi-classical perturbations of the Euclidean Laplace-Beltrami operator. We show that if the suitably cut-off resolvent of the Hamiltonian quantizes a Lagrangian relation on the product cotangent bundle, the scattering amplitude quantizes the natural scattering relation. In the case when the resolvent is tempered, which is true under some non-resonance assumptions, and when we work microlocally near a non-trapped ray our result implies that the scattering amplitude defines a semiclassical Fourier integral operator associated to the scattering relation in a neighborhood of that ray. Compared to previous work we allow this relation to have more general geometric structure.
\end{abstract}

\section{Introduction and Statement of Results}

We study the semi-classical scattering amplitude at non-trapping energies for compactly supported metric and potential perturbation $P(h)$ of the Euclidean Laplace-Beltrami operator $P_{0}(h)$ on $\mathbb{R}^{n}$. The scattering amplitude at energy $\lambda>0$ is the amplitude of the leading term in the asymptotic expansion of an outgoing solution of $(P(h)-\lambda) u=0$ as $\|x\|=r \rightarrow \infty$. We prove that the scattering amplitude quantizes the natural scattering relation in a sense of global semi-classical Fourier Integral Operators.

\subsection{A Survey of Earlier Results}

The structure of the scattering amplitude has been of considerable interest to researchers in mathematical physics. To outline the earlier results, we begin by making some definitions. Let $P(h)=-\frac{1}{2} h^{2} \Delta+V$, where

$$
\left|\frac{\partial^{\alpha}}{\partial x^{\alpha}} V(x)\right| \leq C_{\alpha}(1+|x|)^{-\mu-|\alpha|}, x \in \mathbb{R}^{n}, \mu>0 .
$$

Let $\omega_{0} \in \mathbb{S}^{n-1}$ and for $z \in \omega_{0}^{\perp}$, with $z$ its coordinate representation in $\mathbb{R}^{n-1}$, let

$$
\left\{q_{\infty}(t ; z, \lambda), p_{\infty}(t ; z, \lambda)\right\}
$$


be the unique phase trajectory such that

$$
\begin{gathered}
\lim _{t \rightarrow-\infty}\left|q_{\infty}(t ; z, \lambda)-\sqrt{2 \lambda} \omega_{0} t-z\right|=0, \\
\lim _{t \rightarrow-\infty}\left|p_{\infty}(t ; z, \lambda)-\sqrt{2 \lambda} \omega_{0}\right|=0
\end{gathered}
$$

in the $C^{\infty}$ topology for the impact parameter $z$. Then there exist $\xi_{\infty}(z ; \lambda), r_{\infty}(z ; \lambda) \in$ $C^{\infty}\left(\omega^{\perp}\right)$ with $\xi_{\infty}(z ; \lambda) \in \mathbb{S}^{n-1}$, such that

$$
\begin{gathered}
\lim _{t \rightarrow \infty}\left|q_{\infty}(t ; z, \lambda)-\sqrt{2 \lambda} \xi_{\infty}(z ; \lambda) t-r_{\infty}(z ; \lambda)\right|=0, \\
\lim _{t \rightarrow \infty}\left|q_{\infty}(t ; z, \lambda)-\sqrt{2 \lambda} \xi_{\infty}(z ; \lambda)\right|=0,
\end{gathered}
$$

in the $C^{\infty}$ topology for $z$. The trajectory $\left\{q_{\infty}(t ; z, \lambda), p_{\infty}(t ; z, \lambda)\right\}$ is then said to have initial direction $\omega_{0}$ and final direction $\theta_{0}=\xi_{\infty}(z, \lambda)$ and $\theta_{0}$ is said to be non-degenerate, or regular, for $\omega_{0}$ if for all $z \in \omega_{0}^{\perp}$ with $\xi_{\infty}(z ; \lambda)=\theta_{0}$, the angular density $\hat{\sigma}(z ; \lambda)$ for the trajectory $\left\{q_{\infty}(t ; z, \lambda), p_{\infty}(t ; z, \lambda)\right\}$ satisfies

$$
\hat{\sigma}(z ; \lambda):=\left|\operatorname{det}\left(\xi_{\infty}, \frac{\partial}{\partial z_{1}} \xi_{\infty}, \ldots, \frac{\partial}{\partial z_{n-1}} \xi_{\infty}\right)\right| \neq 0 .
$$

The first asymptotic expansion of the semi-classical scattering amplitude was given by Vainberg 20. He considers the semi-classical Schrödinger operator with a potential $V \in$ $C_{c}^{\infty}\left(\mathbb{R}^{n} ; \mathbb{R}\right)$ and assumes (2). For the associated scattering amplitude at energies $\lambda>\sup V$ he then proves an asymptotic expansion of the scattering amplitude in the form

$$
f(\theta, \lambda)=\sum_{j=1}^{l} \hat{\sigma}\left(z_{j} ; \lambda\right)^{-1 / 2} \exp \left(i h^{-1} S_{j}-i \mu_{j} \pi / 2\right)+\mathcal{O}(h),
$$

where $\left(z_{j}\right)_{j=1}^{l} \equiv\left(\xi_{\infty}^{-1}(\cdot ; \lambda)\right)\left(\theta_{0}\right), S_{j}$ is a modified action along the $j$-th $\left(\omega_{0}, \theta\right)$ trajectory, $\theta \in U$, where $U \subset \mathbb{S}^{n-1}$ is a sufficiently small open neighborhood of $\theta_{0}$, and $\mu_{j}$ is the path index of the trajectory. The error term is estimated uniformly in $\theta \in U$.

Majda [7] considers scattering processes defined by the classical wave equation in the presence of a convex obstacle in $\mathbb{R}^{n}$ where $n=2,3$. For Dirichlet, Neumann, and in the case of three-dimensional space, impedance boundary conditions, he proves an asymptotic expansion of the scattering amplitude, the leading term of which is the product of the Gauss curvature and the reflection coefficient evaluated at a point on the boundary of the obstacle. In this setting the scattering amplitude is the coefficient of the leading term in the asymptotic expansion of an outgoing solution of the reduced wave equation. To establish the aforementioned result, he studies the radiation pattern of a solution which approximates this outgoing solution near the boundary of the obstacle. He also applies his main result to inverse scattering problems for convex bodies with the above boundary conditions. In particular, he proves that both the shape of the boundary of the obstacle and the nature of 
the boundary conditions are completely determined by the asymptotic limit of the scattering amplitude.

Guillemin [5] discusses similar asymptotic expansions. He studies the behavior of the scattering matrix in several different settings: on a compact manifold, in obstacle scattering, for a compactly supported perturbation of the Euclidean metric on $\mathbb{R}^{n}$, and for a quotient Riemannian manifold. In each case, he presents formulas for the kernel of the scattering matrix at energy $\lambda$ of the form

$$
S(\lambda, \omega, \theta)=c(\lambda, n) \sum_{j=1}^{N} c(j)\left|J_{j}(\lambda)\right|^{-1 / 2} e^{i \lambda T_{j}}+\mathcal{O}\left(\frac{1}{\lambda}\right), \theta \neq \omega
$$

under the assumption that there are $N$ scattering rays with initial direction $\omega$ and final direction $\theta$, where $T_{j}$ is the sojourn time of the $j$-th scattering ray and $J_{j}$ is the scattering differential cross-section evaluated at the point of incidence of the $j$-th scattering ray. In the case of the quotient, the scattering matrix is a unitary matrix of size depending on the topology of the manifold. For each energy level $\lambda$ its $j k$-th entry has the form

$$
S_{j k}(\lambda)=a c(\lambda) \sum e^{-T_{k}(i \lambda+1 / 2)}
$$

where

$$
c(\lambda)=\int_{-\infty}^{\infty} \frac{d q}{\left(1+q^{2}\right)^{1 / 2+i \lambda}} .
$$

To derive these results, Guillemin uses the representation of the scattering operator in terms of the wave operators. He also derives a formula for the scattering differential crosssection in the case of scattering by a smooth convex obstacle from which he deduces that the asymptotic behavior of the scattering amplitude determines the shape of the scatterer.

A different form of the asymptotic expansion of the scattering matrix was given by Protas [15]. He works in the setting considered by Vainberg [20] and proves an asymptotic expansion of the scattering amplitude in terms of canonical Maslov operators. This expansion holds for a fixed initial direction and uniformly in an open set containing the final direction and disjoint from the initial direction.

Yajima 22] was the first to prove an asymptotic expansion of the form (3) of the scattering amplitude for potential perturbations $V \in C^{\infty}\left(\mathbb{R}^{n} ; \mathbb{R}\right)$ of the semi-classical Laplacian satisfying (11) for a constant $\mu>\max \left(1, \frac{n-1}{2}\right)$. He also works at non-trapping energies and with outgoing directions non-degenerate for the initial directions. His results, however, are only valid in the $L^{2}$ sense and under the non-trapping assumption and for outgoing directions which are non-degenerate for the fixed incoming direction.

Robert and Tamura [16] work in the same setting as Yajima 22] with $\mu \geq 1$. For scattering amplitude at non-trapping energies $\lambda>0$, which now satisfies

$$
f(\lambda, h) \in C^{\infty}\left(\mathbb{S}^{n-1} \times \mathbb{S}^{n-1} \backslash \operatorname{diag}\left(\mathbb{S}^{n-1} \times \mathbb{S}^{n-1}\right)\right)
$$


they establish an asymptotic expansion of the form (3) with

$$
S_{j}=\int_{-\infty}^{\infty}\left(\left|p_{\infty}\left(t ; z_{j}, \lambda\right)\right|^{2} / 2-V\left(q_{\infty}\left(t ; z_{j}, \lambda\right)\right)-\lambda\right) d t-\left\langle r_{\infty}\left(w_{j} ; \lambda\right), \sqrt{2 \lambda} \theta\right\rangle .
$$

Michel [9] works in the same setting as Robert and Tamura but he allows the energy level to be trapping while satisfying the condition

$$
\begin{aligned}
& \text { There exists a neighborhood } W \text { of } \omega_{0} \in \mathbb{S}^{n-1} \text { such } \\
& \text { that } \forall \omega^{\prime} \in W, \forall z \in\left(\omega^{\prime}\right)^{\perp} \lim _{t \rightarrow \infty}\left|q_{\infty}\left(t, z, \omega^{\prime}\right)\right|=\infty \text {. }
\end{aligned}
$$

He further assumes that there exists $\epsilon>0$ such that the resonances $\lambda_{j}$ satisfy $\left|\Im \lambda_{j}\right| \geq C h^{q}$ for $\Re \lambda_{j} \in[\lambda-\epsilon, \lambda+\epsilon]$. Under these assumptions he establishes the same asymptotic expansion of the scattering amplitude (3). Like Robert and Tamura, Michel also uses Isozaki-Kitada's representation formula of the scattering amplitude.

\subsection{Statement of Main Theorem}

In this article we analyze the semi-classical scattering amplitude from a different point of view and without making any geometric assumptions on the scattering relation such as (2). We show that under a microlocal assumption on the resolvent, essentially the assumption that a suitably cut-off resolvent quantizes the flow relation, the scattering amplitude is a semi-classical Fourier Integral Operator associated to the classical scattering relation. In other words, it quantizes that canonical relation.

We will work in the abstract "black box" framework of [17 which means that we can formulate our hypotheses independently of the structure of the scatterer. We refer to Section 2.2 for the definition of the scattering amplitude $A(\lambda, h)$, and to [1, Section 3.2] for a complete characterization of the class of semi-classical Fourier integral distributions $I_{h}$. We also review briefly the definition of semi-classical Fourier integral distributions in Section 2.1. The notion of microlocal localization is also reviewed in Section 2.1]

To state our main theorem, we also let

$$
\pi_{1}: T^{*} \mathbb{S}^{n-1} \times T^{*} \mathbb{S}^{n-1} \times T^{*} \mathbb{R}^{n} \times T^{*} \mathbb{R}^{n} \rightarrow T^{*} \mathbb{S}^{n-1} \times T^{*} \mathbb{S}^{n-1}
$$

and

$$
\pi_{2}: T^{*} \mathbb{S}^{n-1} \times T^{*} \mathbb{S}^{n-1} \times T^{*} \mathbb{R}^{n} \times T^{*} \mathbb{R}^{n} \rightarrow T^{*} \mathbb{R}^{n} \times T^{*} \mathbb{R}^{n}
$$

denote the canonical projections. We further introduce the following Lagrangian submanifold of

$$
T^{*}\left(\mathbb{S}^{n-1} \times \mathbb{S}^{n-1} \times \mathbb{R}^{n} \times \mathbb{R}^{n}\right)
$$

depending on the real energy $\lambda>0$ :

$$
\begin{gathered}
\Lambda(\lambda)=\left\{(m, d f(m)): m \in \mathbb{S}^{n-1} \times \mathbb{S}^{n-1} \times \mathbb{R}^{n} \backslash B\left(0, R_{0}\right) \times \mathbb{R}^{n} \backslash B\left(0, R_{0}\right)\right\} \\
f(m)=-\sqrt{2 \lambda}\langle x, \omega\rangle+\sqrt{2 \lambda}\langle y, \theta\rangle, \quad m=(\omega, \theta, x, y) .
\end{gathered}
$$

Lastly, we make the following two assumptions: 
Assumption 1 There exists $s \in \mathbb{R}$ such that for every $\varphi \in C_{c}^{\infty}\left(\mathbb{R}^{n}\right), \varphi \equiv$ const. on $B\left(0, R_{0}\right),\|\varphi R(\lambda, h) \varphi\|_{\mathcal{B}\left(L^{2}\left(\mathbb{R}^{n}\right)\right)}=\mathcal{O}\left(h^{s}\right)$.

Assumption 2 There exists a Lagrangian submanifold $\Lambda_{R}(\lambda) \subset \pi_{2}(\Lambda(\lambda))$ of $T^{*} \mathbb{R}^{n} \times T^{*} \mathbb{R}^{n}$ such that

$$
\begin{gathered}
\text { for every } \tilde{\chi}_{j} \in C_{c}^{\infty}\left(\mathbb{R}^{n} \backslash B\left(0, R_{0}\right)\right), j=1,2, \operatorname{supp} \tilde{\chi}_{1} \cap \operatorname{supp} \tilde{\chi}_{2}=\emptyset, \\
D K_{\tilde{\chi}_{2} R(\lambda, h) \tilde{\chi}_{1}} \in I_{h}^{r}\left(\mathbb{R}^{n} \times \mathbb{R}^{n}, \Lambda_{R}(\lambda)\right),
\end{gathered}
$$

where $D \in \Psi_{h}^{0}\left(1, \mathbb{R}^{n} \times \mathbb{R}^{n}\right)$ is a microlocal cut-off to a neighborhood of

$$
\left\{\left(x_{0}+t \omega_{0}, y_{0}+s \theta_{0}, \sqrt{2 \lambda} \omega_{0},-\sqrt{2 \lambda} \theta_{0}\right): s \in\left[s_{1}, s_{2}\right], t \in\left[t_{1}, t_{2}\right]\right\},
$$

for some fixed $s_{1}<s_{2}<0, t_{1}>t_{2}>0, \omega_{0}, \theta_{0}, x_{0}$, and $y_{0}$, where $K_{\tilde{\chi}_{2} R(\lambda, h) \tilde{\chi}_{1}}$ denotes the Schwartz kernel of the cut-off resolvent.

The last assumption means that the cut-off resolvent is a Fourier Integral Operator microlocally near some incoming and outgoing directions. The first assumption is made so that the notion of applying semi-classical pseudodifferential operators (which here are defined up to residual terms in $\left.\mathcal{O}\left(h^{\infty}\right)\right)$ makes sense. It will be used explicitly in the discussion of the resolvent near a non-trapped trajectory in Sections 5.2 and 5.3.

We also note that implicit in our assumptions is the fact that $\lambda$ is non-resonant, in the sense that the resolvent does not have a pole at $\lambda$.

We can now state our

Main Theorem. Suppose that Assumptions 1 and 2 hold.

Then

$$
\pi_{1} \circ\left(\left.\pi_{2}\right|_{\Lambda(\lambda)}\right)^{-1} \Lambda_{R}(\lambda)
$$

is a smooth Lagrangian submanifold of $T^{*} S^{n-1} \times T^{*} S^{n-1}$ near

$$
\bar{p}=\left(\omega_{0},-\sqrt{2 \lambda} d_{\omega}\left\langle x_{0}, \omega_{0}\right\rangle ; \theta_{0}, \sqrt{2 \lambda} d_{\theta}\left\langle y_{0}, \theta_{0}\right\rangle\right)
$$

and for every $C \in \Psi_{h}^{0}\left(1, \mathbb{S}^{n-1} \times \mathbb{S}^{n-1}\right)$ with symbol supported near $\bar{p}$ we have

$$
C K_{A(\lambda, h)} \in I_{h}^{r+\frac{1}{2}}\left(\mathbb{S}^{n-1} \times \mathbb{S}^{n-1}, \pi_{1} \circ\left(\left.\pi_{2}\right|_{\Lambda(\lambda))}\right)^{-1}\left(\Lambda_{R}(\lambda)\right)\right),
$$

where $K_{A(\lambda, h)}$ denotes the Schwartz kernel of the scattering amplitude.

In the special case when the non-degeneracy assumption (2) holds we recover the phases (41) in (3) - see Theorem [5 below. We expect that a finer analysis based on our method would give a precise description of amplitudes. What is different here is the fact that we can handle the cases in which the scattering relation cannot be parameterized simply. That always occurs at the transition between the perturbation and free propagation - see Figure 1. 


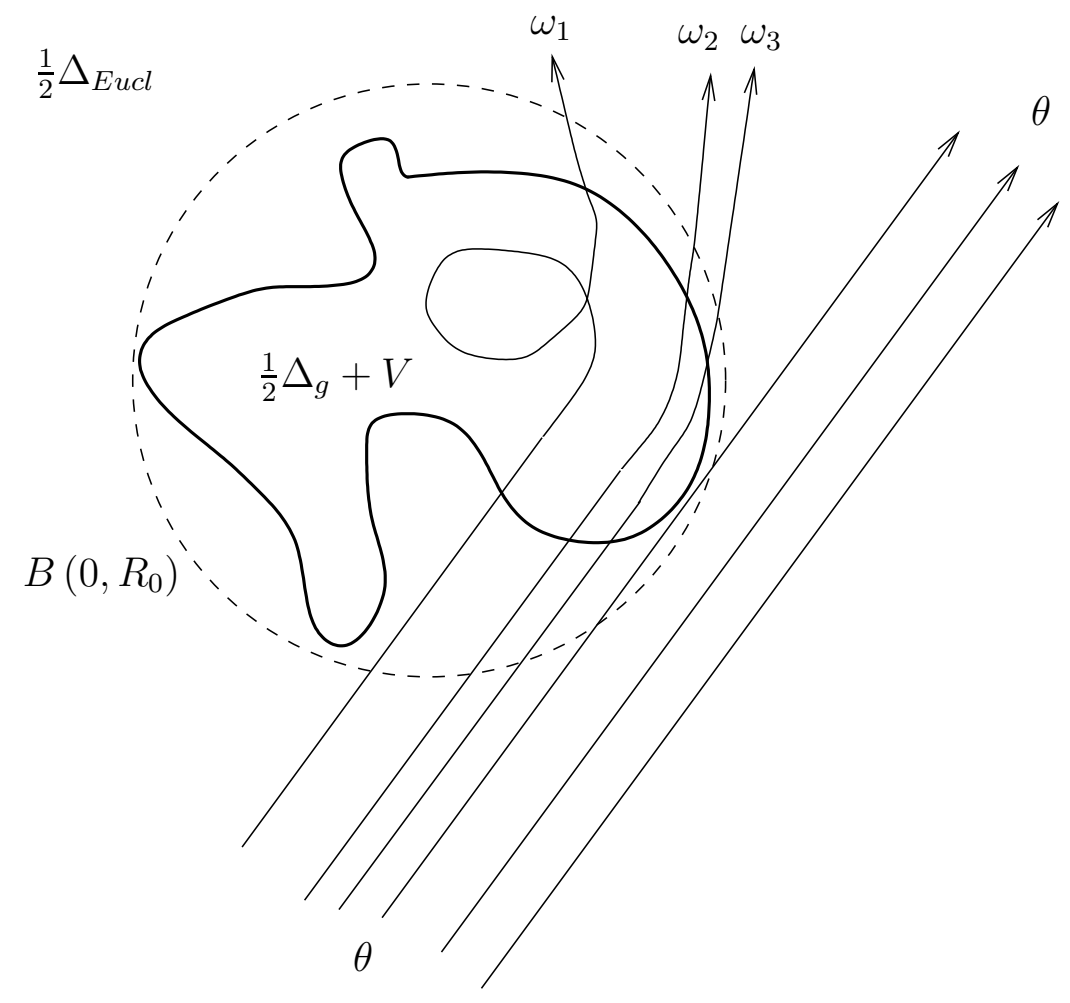

Figure 1: A typical trajectory in the presence of a perturbation. At the boundary between the perturbed trajectories and free trajectories the scattering relation has degenerate projections to the $(\theta, \omega)$ variables.

This paper is organized as follows. In Section 1.3 we introduce some of the notation, which we will use throughout this article. We review the relevant part of semi-classical analysis in Section 2.1. The representation of the scattering matrix, which we will use here, is given in Section [2.2. Section 3 is dedicated to the geometric aspects of the problem with the scattering relation defined and studied in Section 3.1. and the resolvent relation, i. e., the canonical relation which we will prove is quantized by the cut-off resolvent, described in Section 3.2. The proof of the main theorem is given in Section 4. In Section 5 we discuss applications of our main theorem to non-trapping (Section 5.2) and trapping (Section 5.3) smooth compactly supported perturbations of the Euclidean Laplace-Beltrami operator. For that, we prove, in Section [5.1 that the cut-off resolvent for such perturbations satisfies assumption 2. The microlocal representation of the scattering amplitude analogous to (3) under the non-degeneracy assumption (2) on the angular density is given in Section 5.4. Our results are applied to an inverse problem in Section 5.2.1. 


\subsection{Notation}

In this section we introduce some of the notation which we will use below. We shall denote the Euclidean norm on $\mathbb{R}^{n}$, by $\|\cdot\|$ and we set $B(0, r)=\left\{x \in \mathbb{R}^{n} \mid\|x\| \leq r\right\}$ for $r>0$. On any smooth manifold $M$ we denote by $\sigma$ the canonical symplectic form on $T^{*} M$ and everywhere below we work with the canonical symplectic structure on $T^{*} M$. The canonical symplectic coordinates on $T^{*} \mathbb{R}^{n}$ will be denoted by $(x, \xi)$. For a function $f \in C^{\infty}\left(T^{*} M\right)$ we shall denote by $H_{f}$ its Hamiltonian vector field. The integral curve of $H_{f}$ with initial conditions $\left(x_{0}, \xi_{0}\right)$ will be denoted by $\gamma\left(\cdot ; x_{0}, \xi_{0}\right)=\left(x\left(\cdot ; x_{0}, \xi_{0}\right), \xi\left(\cdot ; x_{0}, \xi_{0}\right)\right)$. If $C \subset T^{*} M_{1} \times T^{*} M_{2}$, where $M_{j}$, $j=1,2$, are smooth manifolds we will use the notation $C^{\prime}=\{(x, \xi ; y,-\eta):(x, \xi ; y, \eta) \in C\}$. The Euclidean norm on $\mathbb{R}^{n}$ will be denoted by $\|\cdot\|$ and we set $B(a, R)=\left\{x \in \mathbb{R}^{n} \mid\|a-x\|<\right.$ $R\}$, for $R>0$. For a sequentially continuous operator $T: C_{c}^{\infty}\left(\mathbb{R}^{m}\right) \rightarrow \mathcal{D}^{\prime}\left(\mathbb{R}^{n}\right)$ we shall denote by $K_{T}$ its Schwartz kernel. For such an operator $T$ we use $T^{t}$ to denote the operator with Schwartz kernel $K_{T^{t}}(x, y)=K_{T}(y, x)$. Unless otherwise specified, we will use $\langle\cdot, \cdot\rangle$ to denote the standard inner product on $\mathbb{R}^{n}, \mathbb{C}^{n}$, and $L^{2}\left(\mathbb{R}^{n}\right)$, and $C$ to denote a positive constant, which will be allowed to change from line to line.

\section{Preliminaries}

In this section we present some of the preliminary results we shall use throughout this work.

\subsection{Elements of Semi-Classical Analysis}

In this section we recall some of the elements of semi-classical analysis which we will use here. First we define two classes of symbols

$$
S_{2 n}^{m}(1)=\left\{a \in C^{\infty}\left(\mathbb{R}^{2 n} \times\left(0, h_{0}\right]\right): \forall \alpha, \beta \in \mathbb{N}^{n}, \sup _{(x, \xi, h) \in \mathbb{R}^{2 n} \times\left(0, h_{0}\right]} h^{m}\left|\partial_{x}^{\alpha} \partial_{\xi}^{\beta} a(x, \xi ; h)\right| \leq C_{\alpha, \beta}\right\}
$$

and

$S^{m, k}\left(T^{*} \mathbb{R}^{n}\right)=\left\{a \in C^{\infty}\left(T^{*} \mathbb{R}^{n} \times\left(0, h_{0}\right]\right): \forall \alpha, \beta \in \mathbb{N}^{n},\left|\partial_{x}^{\alpha} \partial_{\xi}^{\beta} a(x, \xi ; h)\right| \leq C_{\alpha, \beta} h^{-m}\langle\xi\rangle^{k-|\beta|}\right\}$,

where $h_{0} \in(0,1]$ and $m, k \in \mathbb{R}$. For $a \in S_{2 n}(1)$ or $a \in S^{m, k}\left(T^{*} \mathbb{R}^{n}\right)$ we define the corresponding semi-classical pseudodifferential operator of class $\Psi_{h, t}^{m}\left(1, \mathbb{R}^{n}\right)$ or $\Psi_{h, t}^{m, k}\left(\mathbb{R}^{n}\right)$, respectively, by setting

$$
O p_{h}(a) u(x)=\frac{1}{(2 \pi h)^{n}} \iint e^{\frac{i\langle x-y, \xi\rangle}{h}} a(x, \xi ; h) u(y) d y d \xi, u \in \mathcal{S}\left(\mathbb{R}^{n}\right),
$$

for $t \in[0,1]$ and extending the definition to $\mathcal{S}^{\prime}\left(\mathbb{R}^{n}\right)$ by duality (see [4]). Below we shall work only with symbols which admit asymptotic expansions in $h$ and with pseudodifferential operators which are quantizations of such symbols. For $A \in \Psi_{h, t}^{k}\left(1, \mathbb{R}^{n}\right)$ or $A \in \Psi_{h, t}^{m, k}\left(\mathbb{R}^{n}\right)$, we 
shall use $\sigma_{0}(A)$ and $\sigma(A)$ to denote its principal symbol and its complete symbol, respectively. A semi-classical pseudodifferential operator will be called of principal type if its principal symbol $a_{0}$ satisfies

$$
a_{0}=0 \Longrightarrow d a_{0} \neq 0 \text {. }
$$

We also define the class of semi-classical distributions $\mathcal{D}_{h}^{\prime}\left(\mathbb{R}^{n}\right)$ with which we will work here

$$
\begin{gathered}
\mathcal{D}_{h}^{\prime}\left(\mathbb{R}^{n}\right)=\left\{u \in C_{h}^{\infty}\left((0,1] ; \mathcal{D}^{\prime}\left(\mathbb{R}^{n}\right)\right): \forall \chi \in C_{c}^{\infty}\left(\mathbb{R}^{n}\right) \exists N \in \mathbb{N} \text { and } C_{N}>0:\right. \\
\left.\left|\mathcal{F}_{h}(\chi u)(\xi, h)\right| \leq C_{N} h^{-N}\langle\xi\rangle^{N}\right\}
\end{gathered}
$$

where

$$
\mathcal{F}_{h}(u)(\xi, h)=\int_{\mathbb{R}^{n}} e^{-\frac{i}{h}\langle x, \xi\rangle} u(x, h) d x
$$

with the obvious extension of this definition to $\mathcal{E}_{h}^{\prime}\left(\mathbb{R}^{n}\right)$. We shall work with the $L^{2}-$ based semi-classical Sobolev spaces $H^{s}\left(\mathbb{R}^{n}\right), s \in \mathbb{R}$, which consist of the distributions $u \in \mathcal{D}_{h}^{\prime}\left(\mathbb{R}^{n}\right)$ such that $\|u\|_{H^{s}\left(\mathbb{R}^{n}\right)}^{2}=\frac{1}{(2 \pi h)^{n}} \int_{\mathbb{R}^{n}}\left(1+\|\xi\|^{2}\right)^{s}\left|\mathcal{F}_{h}(u)(\xi, h)\right|^{2} d \xi<\infty$.

We shall say that $u=v$ microlocally near an open set $U \subset T^{*} \mathbb{R}^{n}$, if $P(u-v)=\mathcal{O}\left(h^{\infty}\right)$ in $C_{c}^{\infty}\left(\mathbb{R}^{n}\right)$ for every $P \in \Psi_{h}^{0}\left(1, \mathbb{R}^{n}\right)$ such that

$$
W F_{h}(P) \subset \tilde{U}, \bar{U} \Subset \tilde{U} \Subset T^{*} \mathbb{R}^{n}, \tilde{U} \text { open. }
$$

We shall also say that $u$ satisfies a property $\mathcal{P}$ microlocally near an open set $U \subset T^{*} \mathbb{R}^{n}$ if there exists $v \in \mathcal{D}_{h}^{\prime}\left(\mathbb{R}^{n}\right)$ such that $u=v$ microlocally near $U$ and $v$ satisfies property $\mathcal{P}$.

For open sets $U, V \subset T^{*} \mathbb{R}^{n}$, the operators $T, T^{\prime} \in \Psi_{h}^{m}\left(\mathbb{R}^{n}\right)$ are said to be microlocally equivalent near $V \times U$ if for any $A, B \in \Psi_{h}^{0}\left(\mathbb{R}^{n}\right)$ such that

$$
\begin{gathered}
W F_{h}(A) \subset \tilde{V}, W F_{h}(B) \subset \tilde{U}, \bar{V} \Subset \tilde{V} \Subset T^{*} \mathbb{R}^{n}, \bar{U} \Subset \tilde{U} \Subset T^{*} \mathbb{R}^{n}, \tilde{U}, \tilde{V} \text { open } \\
A\left(T-T^{\prime}\right) B=\mathcal{O}\left(h^{\infty}\right): \mathcal{D}_{h}^{\prime}\left(\mathbb{R}^{n}\right) \rightarrow C^{\infty}\left(\mathbb{R}^{n}\right) .
\end{gathered}
$$

We shall also use the notation $T \equiv T^{\prime}$.

Lastly, we define global semi-classical Fourier integral operators.

Definition 1 Let $M$ be a smooth $k$-dimensional manifold and let $\Lambda \subset T^{*} M$ be a smooth closed Lagrangian submanifold with respect to the canonical symplectic structure on $T^{*} M$. Let $r \in \mathbb{R}$. Then the space $I_{h}^{r}(M, \Lambda)$ of semi-classical Fourier integral distributions of order $r$ associated to $\Lambda$ is defined as the set of all $u \in \mathcal{D}_{h}^{\prime}(M)$ such that

$$
\left(\prod_{j=0}^{N} A_{j}\right)(u)=\mathcal{O}_{L^{2}(M)}\left(h^{N-r-\frac{k}{4}}\right), h \rightarrow 0,
$$

for all $N \in \mathbb{N}_{0}$ and for all $A_{j} \in \Psi_{h}^{0}(1, X), j=0, \ldots, N-1$, with compactly supported symbols and principal symbols vanishing on $\Lambda$, and any $A_{N} \in \Psi_{h}^{0}(1, X)$ with a compactly supported symbol.

A continuous linear operator $C_{c}^{\infty}\left(M_{1}\right) \rightarrow \mathcal{D}_{h}^{\prime}\left(M_{2}\right)$, where $M_{1}, M_{2}$ are smooth manifolds, whose Schwartz kernel is an element of $I_{h}^{r}\left(M_{1} \times M_{2}, \Lambda\right)$ for some Lagrangian submanifold $\Lambda \subset T^{*} M_{1} \times T^{*} M_{2}$ and some $r \in \mathbb{R}$ will be called a global semi-classical Fourier integral operator of order $r$ associated to $\Lambda$. We denote the space of these operators by $\mathcal{I}_{h}^{r}\left(M_{1} \times M_{2}, \Lambda\right)$. 


\subsection{Representation of the Scattering Amplitude}

In this section we define the semi-classical scattering amplitude and derive the representation of the scattering amplitude, which will be used in the proof of Theorem 1. The derivation is similar to the one presented in [18].

We recall that for any $\theta \in \mathbb{S}^{n-1}$ and $\lambda>0$ there exists a unique, up to a compactly supported function, solution $\psi$ to the problem $(P(h)-\lambda) \psi(\cdot, \theta ; \lambda, h)=0, \psi(\cdot, \theta ; \lambda, h) \in$ $\mathcal{D}_{\text {loc }}(P(h))$ such that

$$
\psi(x, \theta ; \lambda, h)=(1-\chi(x)) e^{\frac{i \sqrt{2 \lambda} \theta \cdot x}{h}}+\psi_{s c}(x, \theta ; \lambda, h),
$$

where $\psi_{s c}$ satisfies the Sommerfeld outgoing condition at infinity:

$$
(\partial / \partial r-i \sqrt{2 \lambda} / h) \psi_{s c}=\mathcal{O}\left(r^{-(n+1) / 2}\right), \text { as } r=\|x\| \rightarrow \infty
$$

and $\chi \in C_{c}^{\infty}\left(\mathbb{R}^{n}\right)$ is equal to 1 on $B\left(0, R_{0}\right)$. Then

$$
\psi(x, \theta ; \lambda, h)=e^{\frac{i \sqrt{2 \lambda} \theta \cdot x}{h}}+\frac{e^{i \sqrt{2 \lambda} r / h}}{r^{(n-1) / 2}} A\left(\frac{x}{r}, \theta ; \lambda, h\right)+\mathcal{O}\left(\frac{1}{r^{(n+1) / 2}}\right) \text {, as } r=\|x\| \rightarrow \infty .
$$

The function $A$ is called the scattering amplitude.

Let, now, $\chi_{1} \in C_{c}^{\infty}\left(\mathbb{R}^{n}\right)$ be equal to 1 on $B\left(0, R_{0}\right)$ and let $\chi_{2} \in C_{c}^{\infty}\left(\mathbb{R}^{n}\right)$ be equal to 1 the support of $\chi_{1}$. Let

$$
\psi=\left(1-\chi_{1}\right) e^{\frac{i \sqrt{2 \lambda}\langle\theta, \cdot\rangle}{h}}+\psi_{s c}
$$

be such that

$$
(P(h)-\lambda) \psi=0 .
$$

Then

$$
\begin{aligned}
(P(h)-\lambda) \psi_{s c} & =-(P(h)-\lambda)\left(1-\chi_{1}\right) e^{\frac{i \sqrt{2 \lambda}(\theta, \cdot\rangle}{h}} \\
& =-\left(-\frac{1}{2} h^{2} \Delta-\lambda\right)\left(1-\chi_{1}\right) e^{\frac{i \sqrt{2 \lambda}\langle\theta, \cdot}{h}}=-\frac{1}{2}\left[h^{2} \Delta, \chi_{1}\right] e^{\frac{i \sqrt{2 \lambda}\langle\theta, \cdot}{h}}
\end{aligned}
$$

and therefore

$$
\psi_{s c}=-\frac{1}{2} R(\lambda, h)\left[h^{2} \Delta, \chi_{1}\right] e^{\frac{i \sqrt{2 \lambda}(\theta, \cdot)}{h}} .
$$

Then

$$
\left(-\frac{1}{2} h^{2} \Delta-\lambda\right)\left(1-\chi_{2}\right) \psi_{s c}=\frac{1}{2}\left[h^{2} \Delta, \chi_{2}\right] \psi_{s c}+\left(1-\chi_{2}\right)(P(h)-\lambda) \psi_{s c}
$$

Substituting (8) into (10), we obtain

$$
\begin{aligned}
\left(-\frac{1}{2} h^{2} \Delta-\lambda\right)\left(1-\chi_{2}\right) \psi_{s c} & =\frac{1}{2}\left[h^{2} \Delta, \chi_{2}\right] \psi_{s c}-\frac{1}{2}\left(1-\chi_{2}\right)\left[h^{2} \Delta, \chi_{1}\right] e^{\frac{i \sqrt{2 \lambda}\langle\theta \cdot \cdot\rangle}{h}} \\
& =\frac{1}{2}\left[h^{2} \Delta, \chi_{2}\right] \psi_{s c}
\end{aligned}
$$


since supp $\nabla \chi_{1} \cap \operatorname{supp}\left(1-\chi_{2}\right)=\emptyset$. Substituting (9) into (11), we obtain

$$
\left(1-\chi_{2}\right) \psi_{s c}=-\frac{1}{4} R_{0}(z, h)\left[h^{2} \Delta, \chi_{2}\right] R(z, h)\left[h^{2} \Delta, \chi_{1}\right] e^{\frac{i \sqrt{2 \lambda}(\theta, \cdot)}{h}} .
$$

Therefore, by Proposition 1.1 in [8], we obtain that the scattering amplitude $A$ is

$$
A(\omega, \theta ; \lambda, h)=c(n, \lambda ; h) \int e^{-\frac{i \sqrt{2 \lambda}\langle\omega, x\rangle}{h}}\left[h^{2} \Delta, \chi_{2}\right] R(\lambda, h)\left[h^{2} \Delta, \chi_{1}\right] e^{\frac{i \sqrt{2 \lambda}\langle\theta \cdot \cdot\rangle}{h}} d x,
$$

where $c(n, \lambda, h)=\frac{e^{-i \pi \frac{n-3}{4}} \lambda^{\frac{n-3}{4}}}{2^{\frac{n+13}{4}}(\pi h)^{\frac{n+1}{2}}}$ and this is the representation of the scattering amplitude, which we will use in the proof of the main theorem. The independence of this representation of the choice of the functions $\chi_{j}, j=1,2$ with the above properties is proved in [14. Proposition 2.1 in [14] further shows that the scattering amplitude with the constant $c(n, \lambda, h)$ replaced by $\tilde{c}(n, \lambda, h)=\frac{i \pi \lambda^{\frac{n-2}{2}}}{(2 \pi)^{n} h^{n}}$ is also the kernel of $S(\lambda, h)-I$, where $S(\lambda, h)$ is the scattering matrix at energy $\lambda$.

From this representation of the scattering amplitude it is clear that it can be extended meromorphically everywhere where the resolvent can be extended meromorphically and that the poles of the scattering amplitude are among the resonances.

We shall now prove two lemmas which give further information about the structure of the cut-off resolvent and the scattering amplitude.

Lemma 1 For $\lambda>0$ not a resonance $K_{R(\lambda, h)}(x, y)=K_{R(\lambda, h)}(y, x)$ for $x, y \in \mathbb{R}^{n} \backslash B\left(0, R_{0}\right)$.

Proof: For $u, v \in L^{2}\left(\mathbb{R}^{n} \backslash B\left(0, R_{0}\right)\right)$ let $\langle u, v\rangle=\int u v$. Let $u$ and $v$ be further chosen with compact support and let $z \in \mathbb{C}$ be such that $\Im z>0$. We then have

$$
\begin{aligned}
\langle( & \left.R(z, h) u)\left.\right|_{\mathbb{R}^{n} \backslash B\left(0, R_{0}\right)}, v\right\rangle=\left\langle\left.(R(z, h) u)\right|_{\mathbb{R}^{n} \backslash B\left(0, R_{0}\right)},(P(h)-z) R(z, h) v\right\rangle \\
& =\left\langle\left.(R(z, h) u)\right|_{\mathbb{R}^{n} \backslash B\left(0, R_{0}\right)},\left(-\frac{1}{2} h^{2} \Delta-z\right)\left(\left.(R(z, h) v)\right|_{\mathbb{R}^{n} \backslash B\left(0, R_{0}\right)}\right)\right\rangle \\
& =\left\langle\left(-\frac{1}{2} h^{2} \Delta-z\right)\left(\left.(R(z, h) u)\right|_{\mathbb{R}^{n} \backslash B\left(0, R_{0}\right)}\right),\left.(R(z, h) v)\right|_{\mathbb{R}^{n} \backslash B\left(0, R_{0}\right)}\right\rangle \\
& =\left\langle\left.((P(h)-z)(R(z, h) u))\right|_{\mathbb{R}^{n} \backslash B\left(0, R_{0}\right)},\left.(R(z, h) v)\right|_{\mathbb{R}^{n} \backslash B\left(0, R_{0}\right)}\right\rangle \\
& =\left\langle u,\left.(R(z, h) v)\right|_{\mathbb{R}^{n} \backslash B\left(0, R_{0}\right)}\right\rangle .
\end{aligned}
$$

Let, now, $\lambda \in \mathbb{R} \backslash\{0\}$ and let $\left(z_{k}\right)_{k \in \mathbb{N}} \subset \mathbb{C}$ satisfy $\Im z_{n}>0, z_{k} \rightarrow \lambda, k \rightarrow \infty$. Then, from (12) we have that for every $k$

$$
\left\langle\left.\left(R\left(z_{k}, h\right) u\right)\right|_{\mathbb{R}^{n} \backslash B\left(0, R_{0}\right)}, v\right\rangle=\left\langle u,\left.\left(R\left(z_{k}, h\right) v\right)\right|_{\mathbb{R}^{n} \backslash B\left(0, R_{0}\right)}\right\rangle .
$$

Letting $k \rightarrow \infty$ in (13) and using the fact that $R(\cdot, h): \mathcal{H}_{c o m p} \rightarrow \mathcal{H}_{l o c}$ is analytic in the upper-half plane and up to the real axis, we obtain

$$
\left\langle\left.(R(\lambda, h) u)\right|_{\mathbb{R}^{n} \backslash B\left(0, R_{0}\right)}, v\right\rangle=\left\langle u,\left.(R(\lambda, h) v)\right|_{\mathbb{R}^{n} \backslash B\left(0, R_{0}\right)}\right\rangle,
$$

which completes the proof of the lemma. 
Lemma 2 The operator $\psi_{1} R(z, h) \psi_{2}$ has a smooth Schwartz kernel $K_{\psi_{1} R(z, h) \psi_{2}}$ when $z$ is not a resonance and $\psi_{j} \in C_{c}^{\infty}\left(\mathbb{R}^{n} \backslash B\left(0, R_{0}\right)\right), j=1,2$, have disjoint supports.

Proof: Let $\varphi \in C^{\infty}\left(\mathbb{R}^{n}\right)$ and consider

$$
\begin{aligned}
& \left(-\frac{1}{2} h^{2} \Delta-z\right) \psi_{1} R(z, h) \psi_{2} \varphi \\
& =\left[-\frac{1}{2} h^{2} \Delta, \psi_{1}\right] R(z, h) \psi_{2} \varphi+\psi_{1}\left(-\frac{1}{2} h^{2} \Delta-z\right)\left(\left.\left(R(z, h) \psi_{2} \varphi\right)\right|_{\mathbb{R}^{n} \backslash B\left(0, R_{0}\right)}\right) \\
& =\left[-\frac{1}{2} h^{2} \Delta, \psi_{1}\right] R(z, h) \psi_{2} \varphi+\left.\psi_{1}\left((P(h)-z) R(z, h) \psi_{2} \varphi\right)\right|_{\mathbb{R}^{n} \backslash B\left(0, R_{0}\right)} \\
& =\left[-\frac{1}{2} h^{2} \Delta, \psi_{1}\right] R(z, h) \psi_{2} \varphi+\psi_{1} \psi_{2} \varphi \\
& =\left[-\frac{1}{2} h^{2} \Delta, \psi_{1}\right] R(z, h) \psi_{2} \varphi \in H^{-1}\left(\mathbb{R}^{n}\right) .
\end{aligned}
$$

Therefore,

$$
\psi_{1} R(z, h) \psi_{2} \varphi \in H^{1}\left(\mathbb{R}^{n}\right) .
$$

Similarly, for every $k \in \mathbb{N}$ we have that

$$
\begin{aligned}
& \left(-\frac{1}{2} h^{2} \Delta-z\right)^{k} \psi_{1} R(z, h) \psi_{2} \varphi \\
& =\underbrace{\left[-\frac{1}{2} h^{2} \Delta,\left[\ldots,\left[-\frac{1}{2} h^{2} \Delta, \psi_{1}\right] \ldots\right]\right]}_{k} R(z, h) \psi_{2} \varphi \in H^{-k}\left(\mathbb{R}^{n}\right)
\end{aligned}
$$

and therefore

$$
\psi_{1} R(z, h) \psi_{2} \varphi \in H^{k}\left(\mathbb{R}^{n}\right) \text { for every } k
$$

Thus

$$
\psi_{1} R(z, h) \psi_{2} \varphi \in C^{\infty}\left(\mathbb{R}^{n}\right) .
$$

This, together with Lemma 1, implies that the kernel of $\psi_{1} R(\lambda, h) \psi_{2}$ is smooth.

As in 14] we, now, introduce the operators

$$
\left[\mathbb{E}_{ \pm}(\lambda, h) f\right](\omega)=\int e^{ \pm \frac{i \sqrt{2 \lambda} \omega \cdot x}{h}} f(x) d x=\hat{f}\left(\mp \frac{\sqrt{2 \lambda} \omega}{h}\right), \omega \in S^{n-1}
$$

where $f$ has compact support. Then we can express $A$ as

$$
A(\lambda, h)=c(n, \lambda, h)\left(\mathbb{E}_{-}(\lambda, h) \otimes \mathbb{E}_{+}(\lambda, h)\right)\left(\left[h^{2} \Delta, \chi_{2}\right] \otimes\left[h^{2} \Delta, \chi_{1}\right]^{t}\right) K_{\tilde{\chi}_{2} R(z, h) \tilde{\chi}_{1}},
$$

where $\tilde{\chi}_{j} \in C_{c}^{\infty}\left(\mathbb{R}^{n} \backslash B\left(0, R_{0}\right)\right)$ are such that $\tilde{\chi}_{j}=1$ on $\operatorname{supp} \nabla \chi_{j}, j=1,2$ and $\operatorname{supp} \tilde{\chi}_{1} \cap$ $\operatorname{supp} \tilde{\chi}_{2}=\emptyset$. 


\section{Scattering Geometry}

Here we collect the geometric results which we will use in the proof of the main theorem and in the applications.

\subsection{Scattering Relation}

Here we define the canonical relation, which we will prove to be quantized by the scattering amplitude in the sense of semi-classical Fourier integral operators. We shall work in the following setting. Let $X$ be a smooth manifold of dimension $n>1$ such that $X$ coincides with $\mathbb{R}^{n}$ outside of $B\left(0, R_{0}\right)$ for some $R_{0}>0$. Let $g$ be a Riemannian metric on $X$ which satisfies the condition

$$
g_{i j}(x)=\delta_{i j} \text { for }\|x\|>R_{0} .
$$

Let $V \in C_{c}^{\infty}\left(X \backslash B\left(0, R_{0}\right)^{c} ; \mathbb{R}\right)$. Let $P(h)=\frac{1}{2} h^{2} \Delta_{g}+V, 0<h \leq 1$, with $p(x, \xi)=\frac{1}{2}\|\xi\|_{g}+$ $V(x)$ denoting its semi-classical principal symbol. We assume that for some $\lambda>0$ the operator $P(h)-\lambda$ is of princiapl type. This implies that $\Sigma_{\lambda}=p^{-1}(\lambda)$ is a smooth $2 n-1$ dimensional manifold. Let $\left(x_{0}, \xi_{0}\right) \in \Sigma_{\lambda}$ with $x_{0} \notin B\left(0, R_{0}\right)^{c}$ be such that there exists $T>0$ satisfying $x\left(t ; x_{0}, \xi_{0}\right) \in B\left(0, R_{0}\right)^{c}$ for all $|t|>T$. The curve $\gamma\left(x_{0}, \xi_{0}\right)$ is called a non-trapped phase trajectory. Then it follows that there exists an open neighborhood $W \subset T^{*} X$ of $\left(x_{0}, \xi_{0}\right)$ such that for all $\left(x^{\prime}, \xi^{\prime}\right) \in W \cap \Sigma_{\lambda}$ we have that $x\left(t ; x^{\prime}, \xi^{\prime}\right) \in B\left(0, R_{0}\right)^{c}$ for all $|t|>T$.

Let, now, $i: T^{*} \mathbb{S}^{n-1} \hookrightarrow T^{*} \mathbb{R}^{n}$ denote the inclusion map. Let $\psi: T^{*} \mathbb{R}^{n} \ni(x, \xi) \mapsto(\xi, x) \in$ $T^{*}\left(\mathbb{R}^{n}\right)^{*}$. Then $L=(\psi \circ i)\left(T^{*} \mathbb{S}^{n-1}\right)$ is a smooth submanifold of $T^{*} \mathbb{R}^{n}$ and therefore

$$
L_{1}(\lambda)=\left\{(x, \xi) \mid\left(x+\frac{\left(1+R_{0}\right)}{\sqrt{2 \lambda}} \xi, \frac{\xi}{\sqrt{2 \lambda}}\right) \in L\right\} \subset \Sigma_{\lambda}
$$

and

$$
L_{2}(\lambda)=\left\{(x, \xi) \mid\left(x+\frac{\left(1-R_{0}\right)}{\sqrt{2 \lambda}} \xi, \frac{\xi}{\sqrt{2 \lambda}}\right) \in L\right\} \subset \Sigma_{\lambda}
$$

are hypersurfaces transverse to $H_{p}$ near $\gamma\left(\cdot ; x_{0}, \xi_{0}\right)$.

Since $\gamma\left(\cdot ; x_{0}, \xi_{0}\right)$ is a non-trapped phase trajectory and since the support of the perturbation is compact, the Hamiltonian flow of $p$ on $\Sigma_{\lambda}$ near $\gamma\left(\cdot ; x_{0}, \xi_{0}\right)$ outside the support of the perturbation is the free Hamiltonian flow defined by the equations

$$
\left\{\begin{array}{l}
\dot{\xi}=0 \\
\dot{x}=\xi
\end{array}\right.
$$

with solution

$$
\left\{\begin{array}{l}
\xi=\sqrt{2 \lambda} \xi_{\infty}=\text { const. } \\
x=\sqrt{2 \lambda} \xi_{\infty} t+x_{\infty}
\end{array}\right.
$$

where by conservation of energy, we have that $\xi_{\infty} \in \mathbb{S}^{n-1}$. 


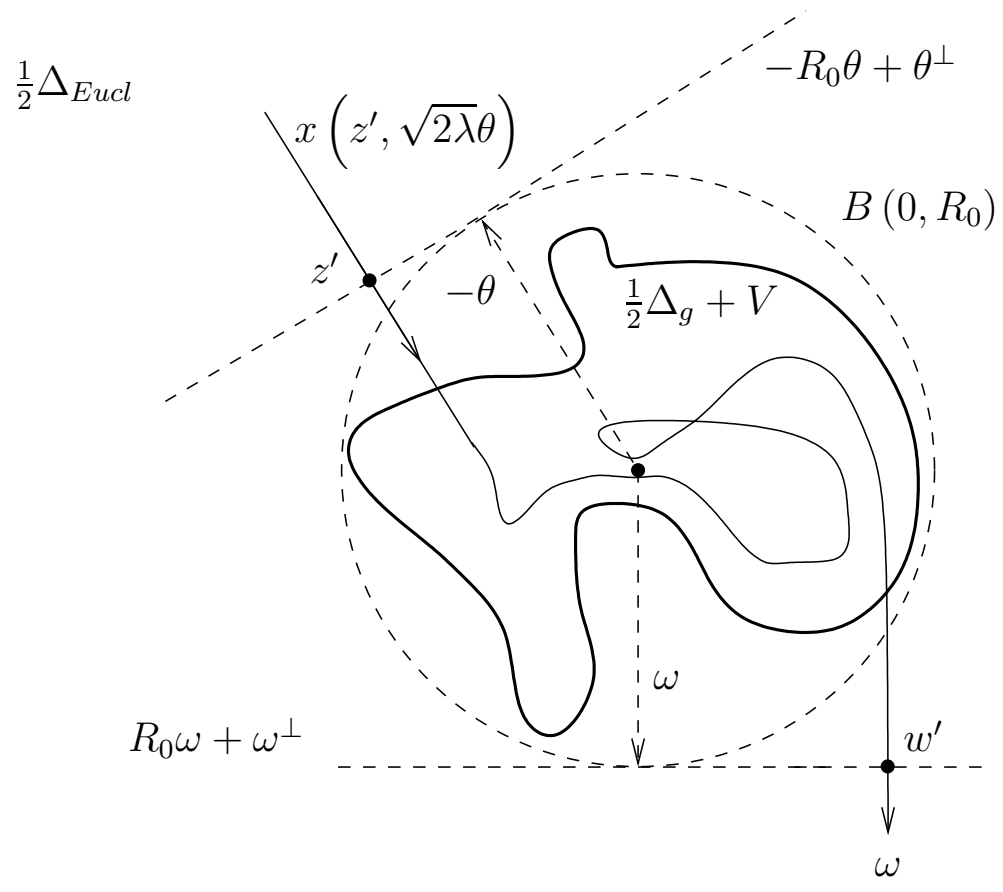

Figure 2: The scattering relation consists of the points $\left(\sqrt{2 \lambda} \theta, z^{\prime} ; \sqrt{2 \lambda} \omega, w^{\prime}\right)$ as in this figure.

Denoting $p_{1}=\gamma\left(\cdot ; x_{0}, \xi_{0}\right) \cap L_{1}(\lambda)$ we then have that for every $(x, \xi) \in L_{1}(\lambda)$ in a sufficiently small neighborhood of $p_{1}$ there exists a unique $T(x, \xi)>0$ such that

$$
\exp \left(T(x, \xi) H_{p}\right)(x, \xi) \in L_{2}(\lambda) .
$$

Therefore, since $L_{1}(\lambda)$ and $L_{2}(\lambda)$ are hypersurfaces in $\Sigma_{\lambda}$ transverse to $H_{p}$ near $\gamma$, we have that there exists an open set $U \subset L_{1}(\lambda), p_{1} \in U$, such that

$$
\begin{aligned}
S R_{U}(\lambda)= & \left\{(x, \xi ; y, \eta) \mid(z, \zeta)=\left(\frac{x}{\sqrt{2 \lambda}}-\left(R_{0}+1\right) \xi, \sqrt{2 \lambda} \xi\right) \in U\right. \\
& \left.\left(\frac{y}{\sqrt{2 \lambda}}+\left(R_{0}-1\right) \eta, \sqrt{2 \lambda} \eta\right)=\exp \left(T(z, \zeta) H_{p}\right)(z, \zeta)\right\}^{\prime}
\end{aligned}
$$

is a Lagrangian submanifold of $\left(T^{*} \mathbb{S}^{n-1} \times T^{*} \mathbb{S}^{n-1}, \pi_{1}^{*} \sigma+\pi_{2}^{*} \sigma\right)$, where $\pi_{j}: T^{*} \mathbb{S}^{n-1} \times T^{*} \mathbb{S}^{n-1} \rightarrow$ $T^{*} \mathbb{S}^{n-1}, j=1,2$, is the projection onto the $j$-th factor. We shall call $S R_{U}(\lambda)$ the scattering relation at energy $\lambda$ (see Figure 2).

We now show how, under a certain geometric assumption, we can find a phase function which parameterizes the scattering relation near a non-trapped trajectory. To state the assumption, let us first introduce some notation. For $\theta \in \mathbb{S}^{n-1}$ and $z \in \theta^{\perp}-R_{0} \theta$, if $\gamma(\cdot ; z, \sqrt{2 \lambda} \theta)$ is a non-trapped trajectory, then, as we saw above, there exist

$$
x_{\infty}(\theta, z), \xi_{\infty}(\theta, z) \in \mathbb{R}^{n},\left\|\xi_{\infty}(\theta, z)\right\|=1,
$$


such that

$$
\gamma(t ; z, \sqrt{2 \lambda} \theta)=\left(x_{\infty}(\theta, z)+t \sqrt{2 \lambda} \xi_{\infty}(\theta, z), \sqrt{2 \lambda} \xi_{\infty}(\theta, z)\right), t>>0 .
$$

We shall call such a non-trapped phase trajectory with initial direction $\theta$ and final direction $\omega=\xi_{\infty}(\theta, z)$, a $(\theta, \omega)$-trajectory. We, now, make the following

Definition 2 If $\theta_{0}, \omega_{0} \in \mathbb{S}^{n-1}$ are such that

$$
\xi_{\infty}\left(\theta_{0}, z^{\prime}\right)=\omega_{0}
$$

implies that the map

$$
\theta_{0}^{\perp}-R_{0} \omega_{0} \ni z \mapsto \xi_{\infty}\left(\theta_{0}, z\right) \in \mathbb{S}^{n-1} \text { is non-degenerate at } z^{\prime},
$$

then we shall say that $\omega_{0}$ is regular for $\theta_{0}$.

We remark that this definition is a rephrasing of condition (2).

We then have the following

Lemma 3 If $\omega_{0} \in \mathbb{S}^{n-1}$ is regular for $\theta_{0} \in \mathbb{S}^{n-1}$, then

(a) $\theta_{0} \neq \omega_{0}$.

(b) There exist $O_{j} \subset \mathbb{S}^{n-1}, j=1,2$, open, $\theta_{0} \in O_{1}, \omega_{0} \in O_{2}$, and a number $L \in \mathbb{N}$ such that for every $(\theta, \omega) \in O_{1} \times O_{2}$, there exist at least $L(\theta, \omega)$ - trajectories.

Proof: We shall work in a local trivialization of $T^{*} \mathbb{S}_{R_{0}}^{n-1}$ near $T_{R_{0} \theta_{0}}^{*} \mathbb{S}_{R_{0}}^{n-1}$ and $T_{R_{0} \omega_{0}}^{*} \mathbb{S}_{R_{0}}^{n-1}$, where $\mathbb{S}_{R_{0}}^{n-1}=\left\{x \in \mathbb{R}^{n}:\|x\|=R_{0}\right\}$. We first prove that $\theta_{0} \neq \omega_{0}$. Assume that $\theta_{0}=\omega_{0}$. Then for every $z \in \mathbb{R}^{n-1}$ with $\|z\|>R_{0}$ we have that $\xi_{\infty}\left(\theta_{0}, z\right)=\theta_{0}$. Therefore $\operatorname{det}\left(\frac{\partial \xi_{\infty}\left(\theta_{0}, \cdot\right)}{\partial z}(z)\right)=0$ for $z \in \theta_{0}^{\perp},\|z\|>R_{0}$, which is a contradiction with the regularity assumption. Thus it follows that $\theta_{0} \neq \omega_{0}$, which establishes (a).

Let, now, $z^{\prime} \in\left(\xi_{\infty}\left(\theta_{0}, \cdot\right)\right)^{-1}\left(\omega_{0}\right)$. Then, by the Inverse Function Theorem, there exist open sets $O_{2}^{\prime} \subset \mathbb{S}^{n-1}, \omega_{0} \in O_{2}^{\prime}$ and $\mathcal{Z}^{\prime} \subset \mathbb{R}^{n-1}, z^{\prime} \in \mathcal{Z}^{\prime}$ such that $\left.\xi_{\infty}\right|_{\mathcal{Z}^{\prime}}\left(\theta_{0}, \cdot\right)$ is a diffeomorphism onto $O_{2}^{\prime}$. Therefore, the set $\left(\xi_{\infty}\left(\theta_{0}, \cdot\right)\right)^{-1}\left(\omega_{0}\right)$ is discrete. From the first part of this proof, it follows that $\left(\xi_{\infty}\left(\theta_{0}, \cdot\right)\right)^{-1}\left(\omega_{0}\right)$ is also bounded. Therefore, it is finite and we shall denote its elements by $\left\{z_{1}, \ldots, z_{L}\right\}, L \in \mathbb{N}$.

By the Implicit Function Theorem and the regularity assumption, we now have that there exist open sets $O_{1}, O_{2} \subset \mathbb{S}^{n-1}$ with $\theta_{0} \in O_{1}$ and $\omega_{0} \in O_{2}$ and functions $z_{l} \in C^{\infty}\left(O_{1} \times\right.$ $\left.O_{2} ; \mathbb{R}^{n-1}\right), l=1, \ldots, L$, such that $z_{l}\left(\theta_{0}, \omega_{0}\right)=z_{l}$ and $\xi_{\infty}\left(\theta, z_{l}(\theta, \omega)\right)=\omega,(\theta, \omega) \in O_{1} \times O_{2}$, which completes the proof of (b).

Let, now, $w_{l}(\theta, \omega)=\gamma\left(\cdot ; z_{l}(\theta, \omega), \sqrt{2 \lambda} \theta\right) \cap\left(R_{0} \omega+\omega^{\perp}\right)$. Then, as above we have that,

$$
\begin{array}{r}
S R_{O_{1} \times O_{2}}^{l}(\lambda)=\left\{\left(\sqrt{2 \lambda} \theta, \sqrt{2 \lambda} \omega, z_{l}(\theta, \omega)+\left(R_{0}-1\right) \theta, w_{l}(\theta, \omega)-\left(R_{0}-1\right) \omega\right) \mid\right. \\
\left.(\theta, \omega) \in O_{1} \times O_{2}\right\}^{\prime}
\end{array}
$$


is a Lagrangian submanifold of $T^{*}\left(\mathbb{S}^{n-1} \times \mathbb{S}^{n-1}\right)$. Furthermore, $\left.\pi\right|_{S R_{O_{1} \times O_{2}}^{l}(\lambda)}$ is a surjection, where $\pi: T^{*}\left(\mathbb{S}^{n-1} \times \mathbb{S}^{n-1}\right) \rightarrow \mathbb{S}^{n-1} \times \mathbb{S}^{n-1}$ is the canonical projection. Therefore, after decreasing, if necessary, $O_{1} \times O_{2}$ around $\left(\theta_{0}, \omega_{0}\right)$ we have that there exists a function $\mathcal{W}_{l} \in$ $C^{\infty}\left(O_{1} \times O_{2}\right)$ such that

$$
S R_{O_{1} \times O_{2}}^{l}(\lambda)=\left\{\left(m, d \mathcal{W}_{l}(m)\right): m \in O_{1} \times O_{2}\right\} .
$$

Since we also have that $S R_{O_{1} \times O_{2}}^{l}(\lambda)$ is a canonical relation and therefore, after possibly decreasing $O_{1} \times O_{2}$ further near $\left(\theta_{0}, \omega_{0}\right)$, we can assume that

$$
\operatorname{det}\left(\partial_{\theta} \partial_{\omega} \mathcal{W}_{l}(\theta, \omega)\right) \neq 0,(\theta, \omega) \in O_{1} \times O_{2}
$$

We let

$$
S R_{l}(\lambda)=S R_{O_{1} \times O_{2}}^{l}(\lambda)
$$

with $O_{1} \times O_{2}$ as in (18). We observe that (18) implies that the map $R_{0} \omega+\omega^{\perp} \ni w \mapsto$ $\xi_{\infty}(-\omega, w) \in \mathbb{S}^{n-1}$ is non-degenerate in a neighborhood of $w_{l}(\theta, \omega),(\theta, \omega) \in O_{1} \times O_{2}$.

For the $(\theta, \omega)$ trajectory defined by $z_{l}(\theta, \omega)$ as in the proof of the Lemma, we shall use the subscript $l$ to distinguish it from all other $(\theta, \omega)$ trajectories.

The same proof as in [16. Lemma 3.2] together with (18) show that there exist $0<S_{0}<S_{1}$ and $T_{0}>>0$ and open sets $U_{\theta, \omega}^{l} \subset \mathbb{R}^{n-1}, z_{l}(\theta, \omega) \in U_{\theta, \omega}^{l},(\theta, \omega) \in O_{1} \times O_{2}, l=1, \ldots, L$ such that

$$
\operatorname{det}\left(\frac{\partial x_{l}(t ; \cdot \sqrt{2 \lambda} \theta)}{\partial y}(y)\right) \neq 0
$$

for $\theta \in O_{1}, y \in\left\{z-s \theta: z \in U_{\theta, \omega}^{l}, s \in\left[S_{0}, S_{1}\right]\right\}, \omega \in O_{2}, t>T_{0}$.

For $(\theta, \omega) \in O_{1} \times O_{2}$ we now define the (modified) action along the segment of the $l$-th $(\theta, \omega)$-trajectory, $\gamma\left(\cdot ; z_{l}(\theta, \omega), \sqrt{2 \lambda} \theta\right)$, between the points $y_{l}(s ; \theta, \omega)=z_{l}(\theta, \omega)-\sqrt{2 \lambda} s \theta \in$ $W_{\theta}, s \in\left[S_{1}, S_{0}\right]$ and $x_{l}(t, s, \theta, \omega)=x_{l}\left(t ; y_{l}(s ; \theta, \omega), \sqrt{2 \lambda} \theta\right), t>T_{0}$. We choose a fixed $t>T_{0}$ and we set

$$
S_{l}(\theta, \omega)=\left\langle y_{l}(s ; \theta, \omega), \sqrt{2 \lambda} \theta\right\rangle+\int_{0}^{t} L\left(x_{l}, \dot{x}_{l}\right) d t-\left\langle x_{l}(t, s, \theta, \omega), \sqrt{2 \lambda} \omega\right\rangle-\lambda t,
$$

where $L(x, \dot{x})=\frac{1}{2}\|\dot{x}\|_{g}^{2}-V(x)$ is the Lagrangian, and the integral is taken over the $l$-th bicharacteristic curve connecting $y_{l}(s ; \theta, \omega)$ and $x_{l}(t, s, \theta, \omega)$. We observe that, since the support of the perturbation is compact, $S_{l}(\theta, \omega)$ is independent of $s$ for $s \in\left[S_{1}, S_{0}\right]$.

Lemma 4 Let $\omega_{0} \in \mathbb{S}^{n-1}$ be regular for $\theta_{0} \in \mathbb{S}^{n-1}$.

Then $S R_{l}(\lambda)=\Lambda_{S_{l}}$, where $\Lambda_{S_{l}}=\left\{\left(\theta, \omega, d_{\theta} S_{l}, d_{\omega} S_{l}\right):(\theta, \omega) \in \mathbb{S}^{n-1} \times \mathbb{S}^{n-1}\right\}, l=1, \ldots, L$.

Proof: 
We consider

$$
\begin{aligned}
d_{\omega} S_{l}(\theta, \omega)= & d_{\omega}\left(\left\langle y_{l}(s ; \theta, \omega), \sqrt{2 \lambda} \theta\right\rangle+\int_{0}^{t} L\left(x_{l}, \dot{x}_{l}\right) d t\right)-d_{\omega}\left(\left\langle x_{l}(t, s, \theta, \cdot), \sqrt{2 \lambda} \cdot\right\rangle\right)(\omega) \\
= & \left\langle\sqrt{2 \lambda} \omega, d_{\omega} x_{l}(t, s, \theta, \cdot)(\omega)\right\rangle-\left\langle\sqrt{2 \lambda} \omega, d_{\omega} x_{l}(t, s, \theta, \cdot)(\omega)\right\rangle \\
& \quad-d_{\omega}\left\langle x_{l}(t, s, \theta, \omega), \sqrt{2 \lambda} \cdot\right\rangle(\omega) \\
= & -d_{\omega}\left\langle x_{l}(\omega, \theta, s, t), \sqrt{2 \lambda} \cdot\right\rangle(\omega),
\end{aligned}
$$

where (20) has allowed us to use [2, Theorem 46.C] to obtain the second equality.

To compute $d_{\theta} S_{l}$ we first reparameterize the phase trajectories in the reverse direction, which is equivalent to considering the reverse of the initial and final directions. We further re-write $S_{l}(\theta, \omega)$ in the following way

$$
S_{l}(\theta, \omega)=-\left\langle x_{l}(s ; \theta, \omega), \sqrt{2 \lambda} \omega\right\rangle+\int_{0}^{t} L\left(x_{l}, \dot{x}_{l}\right) d t+\left\langle y_{l}(t, s, \omega, \theta), \sqrt{2 \lambda} \theta\right\rangle-\lambda t
$$

where $x_{l}(s ; \theta, \omega)=w_{l}(\theta, \omega)+\sqrt{2 \lambda} \omega s, y_{l}(t, s, \theta, \omega)=x_{l}\left(t ; x_{l}(s ; \theta, \omega),-\sqrt{2 \lambda} \omega\right), s \in\left[S_{0}, S_{1}\right]$, and the integral is taken over the bicharacteristic curve connecting $x_{l}(s ; \theta, \omega)$ and $y_{l}(t, s, \theta, \omega)$. We observe that this bicharacteristic curve is uniquely defined by (18) and (20).

Equations (18) and (20) further allow us to proceed as in (22) and we obtain

$$
\begin{aligned}
d_{\theta} S_{l}(\omega, \theta) & =d_{\theta}\left(-\left\langle x_{l}(s ; \theta, \omega), \sqrt{2 \lambda} \omega\right\rangle+\int_{0}^{t} L\left(x_{l}, \dot{x}_{l}\right) d t\right)+d_{\theta}\left(\left\langle y_{l}(t, s, \omega, \theta), \sqrt{2 \lambda} \cdot\right\rangle\right) \\
& =d_{\theta}\left\langle y_{l}(t, s, \theta, \omega), \sqrt{2 \lambda} \cdot\right\rangle(\theta) .
\end{aligned}
$$

From (22) and (23) we therefore have that $S_{l}$ is a non-degenerate phase function such that $S R_{l}(\lambda)=\Lambda_{S_{l}}$.

\subsection{Resolvent Relation}

We now define the Lagrangian submanifold which we will prove is quantized by the cut-off resolvent in the sense of semi-classical Fourier integral operator.

We set

$$
\tilde{\Lambda}_{R}(\lambda, J)=\cup_{t \in J} \text { graph }\left.\exp \left(t H_{p}\right)\right|_{\Sigma_{\lambda}},
$$

where $J \subset \mathbb{R}$ is an open interval. We assume that for every $(x, \xi) \in \Sigma_{\lambda}$ and every $t \in J$, $\exp \left(t H_{p}\right)((x, \xi)) \neq(x, \xi)$. Then $\tilde{\Lambda}_{R}(\lambda, J)$ is a Lagrangian submanifold of $\left(T^{*} \mathbb{R}^{n} \times T^{*} \mathbb{R}^{n}, \tilde{\sigma}\right)$, where $\tilde{\sigma}=\pi_{1}^{*} \sigma-\pi_{2}^{*} \sigma=d \xi \wedge d x-d \eta \wedge d y$, where $\pi_{j}: T^{*} \mathbb{R}^{n} \times T^{*} \mathbb{R}^{n} \rightarrow T^{*} \mathbb{R}^{n}, j=1,2$ is the projection onto the $j$-th factor. We define the resolvent relation as $\Lambda_{R}(\lambda)=\tilde{\Lambda}_{R}^{\prime}\left(\lambda, \mathbb{R}^{+}\right) \cap$ $T^{*}\left(\operatorname{supp} \tilde{\chi}_{2} \times \operatorname{supp} \tilde{\chi}_{1}\right)$. 


\section{Proof of Main Theorem}

We now turn to the proof of our Main Theorem.

Proof: We first prove that the scattering amplitude belongs to $\mathcal{D}_{h}^{\prime}\left(\mathbb{S}^{n-1} \times \mathbb{S}^{n-1}\right)$. For that, let $\psi \in C^{\infty}\left(\mathbb{S}^{n-1} \times \mathbb{S}^{n-1}\right)$ have support in a coordinate chart on $\mathbb{S}^{n-1} \times \mathbb{S}^{n-1}$ with local coordinates $(\tilde{\omega}, \tilde{\theta})$ and let $\psi_{1}, \psi_{2} \in C^{\infty}\left(\mathbb{S}^{n-1}\right)$ be such that $\psi_{1} \times \psi_{2}=1$ on supp $\psi$. Then, since $K_{A(\lambda, h)} \in C^{\infty}\left(\mathbb{S}^{n-1} \times \mathbb{S}^{n-1}\right)$, we have

$$
\begin{aligned}
\left|\mathcal{F}_{h}\left(K_{A(\lambda, h)}\right)(\tilde{\xi}, \tilde{\eta})\right| & =\left|\iint K_{A(\lambda, h)}(\tilde{\omega}, \tilde{\theta}) \psi(\tilde{\omega}, \tilde{\theta}) e^{-\frac{i}{h}(\langle\tilde{\omega}, \tilde{\xi}\rangle+\langle\tilde{\theta}, \tilde{\eta}\rangle)} d \tilde{\theta} d \tilde{\omega}\right| \\
& \leq \iint\left|K_{A(\lambda, h)}(\tilde{\omega}, \tilde{\theta}) \psi(\tilde{\omega}, \tilde{\theta})\right| d \tilde{\theta} d \tilde{\omega} \\
& \leq C \iint\left|K_{A(\lambda, h)}(\tilde{\omega}, \tilde{\theta}) \psi_{1}(\tilde{\omega}) \psi_{2}(\tilde{\theta})\right| d \tilde{\theta} d \tilde{\omega} \\
& \leq C\|A(\lambda, h)\|_{\mathcal{B}\left(L^{2}\left(\mathbb{S}^{n-1}\right)\right)}\left\|\psi_{1}\right\|_{L^{2}\left(\mathbb{S}^{n-1}\right)}\left\|\psi_{2}\right\|_{L^{2}\left(\mathbb{S}^{n-1}\right)} \\
& =\mathcal{O}\left(h^{\frac{1-n}{2}}\right)
\end{aligned}
$$

where the last equality follows from

$$
A(\lambda, h)=h^{\frac{n-1}{2}} \lambda^{\frac{1-n}{4}} 2^{\frac{3 n-13}{4}} \pi^{\frac{n-3}{2}} e^{i \pi \frac{5-n}{4}}(S(\lambda, h)-I)
$$

and the fact that $S(\lambda, h)$ is a unitary operator on $L^{2}\left(\mathbb{S}^{n-1}\right)$.

We also observe that a direct calculation shows that $\pi_{1} \circ\left(\left.\pi_{2}\right|_{\Lambda(\lambda)}\right)^{-1}\left(\Lambda_{R}(\lambda)\right)$ is a Lagrangian submanifold of $T^{*} \mathbb{S}^{n-1} \times T^{*} \mathbb{S}^{n-1}$. To prove the theorem, now, we first note that

$$
\mathbb{E}_{-}(\lambda, h) \otimes \mathbb{E}_{+}(\lambda, h) \in \mathcal{I}_{h}^{-n+\frac{1}{2}}\left(\mathbb{S}^{n-1} \times \mathbb{S}^{n-1} \times \mathbb{R}^{n} \times \mathbb{R}^{n}, \Lambda(\lambda)\right)
$$

and we easily see that

$$
\left.\pi_{2}\right|_{\Lambda(\lambda)} \text { is an immersion. }
$$

Let, now,

$$
A_{j} \in \Psi_{h}^{0}\left(1, \mathbb{S}^{n-1} \times \mathbb{S}^{n-1}\right), j=1, \ldots, N
$$

have symbols supported in a neighborhood of $\bar{p}$ and principal symbols vanishing on $\pi_{1} \circ$ $\left(\left.\pi_{2}\right|_{\Lambda(\lambda)}\right)^{-1}\left(\Lambda_{R}(\lambda)\right)$. From (24) and [1, Lemma 7] we deduce that there exist $D, B_{j} \in$ $\Psi_{h}^{0}\left(1, \mathbb{R}^{2 n}\right), j=1, \ldots, N$, with symbols supported near the point

$$
\bar{q}=\left(x_{0}+t \omega_{0}, y_{0}+s \theta_{0}, \sqrt{2 \lambda} \omega_{0},-\sqrt{2 \lambda} \theta_{0}\right) \text { for some } s \in\left[s_{1}, s_{2}\right], t \in\left[t_{1}, t_{2}\right],
$$

such that

$$
\begin{gathered}
\left(\left.\pi_{2}\right|_{\Lambda(\lambda)}\right)^{*} \sigma_{0}\left(B_{j}\right)=-\left(\left.\pi_{1}\right|_{\Lambda(\lambda)}\right)^{*} \sigma_{0}\left(A_{j}\right), j=1, \ldots, N \\
\left(\left.\pi_{2}\right|_{\Lambda(\lambda)}\right)^{*} \sigma_{0}(D)=-\left(\left.\pi_{1}\right|_{\Lambda(\lambda)}\right)^{*} \sigma_{0}(C)
\end{gathered}
$$


and

$$
\left(\prod_{j=1}^{N} A_{j}\right) C\left(\mathbb{E}_{-}(\lambda, h) \otimes \mathbb{E}_{+}(\lambda, h)\right) \chi \equiv\left(\mathbb{E}_{-}(\lambda, h) \otimes \mathbb{E}_{+}(\lambda, h)\right) \chi \cdot\left(\prod_{j=1}^{N} B_{j}\right) D
$$

near $(\bar{p}, \bar{q})$, where $\chi \in C_{c}^{\infty}\left(\mathbb{R}^{n} \times \mathbb{R}^{n}\right)$ is equal to 1 on supp $\tilde{\chi}_{2} \times \operatorname{supp} \tilde{\chi}_{1}$.

Let, now, $Y=\left[h^{2} \Delta, \chi_{2}\right] \otimes\left[h^{2} \Delta, \chi_{1}\right]^{t}$. Since $Y \in \Psi_{h}^{-1,1}\left(\mathbb{R}^{2 n}\right)$ we obtain from 11, Lemma 5] that

$$
Y K_{\tilde{\chi}_{2} R(\lambda, h) \tilde{\chi}_{1}} \in I_{h}^{r-1}\left(\mathbb{R}^{n} \times \mathbb{R}^{n}, \Lambda_{R}(\lambda)\right) .
$$

Therefore, from (26), we have

$$
\begin{aligned}
& \left(\prod_{j=1}^{N} A_{j}\right) C K_{A(\lambda, h)} \\
& \quad=c(n, \lambda, h)\left(\mathbb{E}_{-}(\lambda, h) \otimes \mathbb{E}_{+}(\lambda, h)\right) \chi \cdot\left(\prod_{j=1}^{N} B_{j}\right) D Y K_{\tilde{\chi}_{2} R(\lambda, h) \tilde{\chi}_{1}}+\mathcal{O}_{L^{2}\left(\mathbb{S}^{n-1} \times \mathbb{S}^{n-1}\right)}\left(h^{\infty}\right) .
\end{aligned}
$$

The choice of the operators $A_{j}$ and (25) now imply

$$
\left.\sigma_{0}\left(B_{j}\right)\right|_{\Lambda_{R}(\lambda)}=0, j=1, \ldots, N .
$$

From [1, Lemma 5] we have again

$$
D Y K_{\tilde{\chi}_{2} R(\lambda, h) \tilde{\chi}_{1}} \in I_{h}^{r-1}\left(\mathbb{R}^{n} \times \mathbb{R}^{n}, \Lambda_{R}(\lambda)\right) .
$$

Therefore,

$$
\left(\prod_{j=1}^{N} B_{j}\right) D Y K_{\tilde{\chi}_{2} R(\lambda, h) \tilde{\chi}_{1}}=\mathcal{O}_{L^{2}\left(\mathbb{R}^{2 n}\right)}\left(h^{N-r+1+\frac{n}{4}}\right) .
$$

Lastly, as in the proof of [3, Proposition 2.1], we have that

$$
\left\|\mathbb{E}_{ \pm}^{\phi}\right\|_{\mathcal{B}\left(L^{2}\left(\mathbb{R}^{n}\right), L^{2}\left(\mathbb{S}^{n-1}\right)\right)}=\mathcal{O}\left(h^{\frac{n-1}{2}}\right)
$$

where $\mathbb{E}_{ \pm}^{\phi}(\lambda, h)$ are the operators with Schwartz kernels

$$
K_{\mathbb{E}_{ \pm}^{\phi}(\lambda, h)}(\omega, x)=\phi(x) \exp ( \pm i \sqrt{2 \lambda}\langle\omega, x\rangle / h)
$$

for $\phi \in C_{c}^{\infty}\left(\mathbb{R}^{n}\right)$.

We now substitute (29) into (28) and use (30) to obtain

$$
\left(\prod_{j=1}^{N} A_{j}\right) C K_{A(\lambda, h)}=\mathcal{O}_{L^{2}\left(\mathbb{R}^{2(n-1)}\right)}\left(h^{N-r-\frac{n}{2}}\right) .
$$

Therefore $C K_{A(\lambda, h)} \in I_{h}^{r+\frac{1}{2}}\left(\mathbb{S}^{n-1} \times \mathbb{S}^{n-1}, \pi_{1}\left(\left.\pi_{2}\right|_{\Lambda(\lambda)}\right)^{-1}\left(\Lambda_{R}(\lambda)\right)\right)$. 


\section{Applications}

Here we discuss two applications of the main theorem to compactly supported potential and metric perturbations of the Euclidean Laplacian. The setting is as follows.

Let the setting be as in Section 3.1. The operators $P(h)=\frac{1}{2} h^{2} \Delta_{g}+V, 0<h \leq 1$, acting on $\mathcal{H}=L^{2}\left(\mathbb{R}^{n}, d \mathrm{vol}_{g}\right)$ and equipped with the common domain $\mathcal{D}=H^{2}\left(\mathbb{R}^{n}, d \operatorname{vol}_{g}\right)$ admit unique self-adjoint extensions, which we denote by the same notation. As before, we denote their resolvents by $R(z, h)=(P(h)-z)^{-1}$ for $z \in \mathbb{C}_{+}$and use the same notation for their meromorphic continuations. Proposition 2.3 in [8] states that there are no resonances in $\mathbb{R} \backslash\{0\}$ in the case of a smooth compactly supported potential perturbation.

We define a non-trapping energy level as follows:

Definition 3 Let $\left\{\left(x\left(\cdot ; x_{0}, \xi_{0}\right), \xi\left(\cdot ; x_{0}, \xi_{0}\right)\right)\right\}$ be the integral curve of $H_{p}$ with initial conditions $\left(x_{0}, \xi_{0}\right)$. The energy $\lambda>0$ is non-trapping if for every $r>0$, there exists $s>0$ such that $\left(x_{0}, \xi_{0}\right) \in \Sigma_{\lambda}$ with $\left\|x_{0}\right\|<r$ implies that $\left\|x\left(s ; x_{0}, \xi_{0}\right)\right\|>r$ for every $|s|>t$. We also introduce the notation $T(r)$ for the infimum over $s$ with this property.

\subsection{The Cut-off Resolvent as a Semi-Classical Fourier Integral Op- erator}

We now prove that the second assumption of our main theorem is satisfied in the setting we have just described.

Theorem 1 Let $\|\chi R(\lambda, h) \chi\|_{\mathcal{B}\left(L^{2}\left(\mathbb{R}^{n}\right)\right)} \leq C_{\chi} h^{s}$ for every $\chi \in C_{c}^{\infty}\left(\mathbb{R}^{n}\right)$ and some $s \in \mathbb{R}$. Let $\rho_{0} \in \Lambda_{R}(\lambda)$ be such that $\gamma\left(\cdot ; \pi_{1}\left(\rho_{0}\right)\right)$ is a non-trapped trajectory.

Then there exists an open set $V \subset \Lambda_{R}(\lambda), \rho_{0} \in V$, such that

$$
\tilde{\chi}_{2} R(\lambda, h) \tilde{\chi}_{1} \in \mathcal{I}_{h}^{1}\left(\mathbb{R}^{2 n}, \bar{V} \cap \Lambda_{R}(\lambda)\right) .
$$

Proof: First, we prove that $K_{\tilde{\chi}_{2} R(\lambda, h) \tilde{\chi}_{1}} \in \mathcal{D}_{h}^{\prime}\left(\mathbb{R}^{2 n}\right)$. For this, let $\chi \in C_{c}^{\infty}\left(\mathbb{R}^{2 n}\right)$ and let $\rho_{j} \in C_{c}^{\infty}\left(\mathbb{R}^{n} \backslash B\left(0, R_{0}\right)\right), j=1,2$, be such that $\rho_{2} \times \rho_{1}=1$ on $\operatorname{supp} \tilde{\chi}_{2} \times \operatorname{supp} \tilde{\chi}_{1} \cap \operatorname{supp} \chi$. Then, since $K_{\tilde{\chi}_{2} R(\lambda, h) \tilde{\chi}_{1}} \in C^{\infty}\left(\mathbb{R}^{2 n}\right)$, we have

$$
\begin{aligned}
\left|\mathcal{F}_{h}\left(K_{\tilde{\chi}_{2} R(\lambda, h) \tilde{\chi}_{1}}\right)(\xi, \eta)\right| & =\left|\iint K_{\tilde{\chi}_{2} R(\lambda, h) \tilde{\chi}_{1}}(x, y) \chi(x, y) e^{-\frac{i}{h}(\langle x, \xi\rangle+\langle y, \eta\rangle)} d x d y\right| \\
& \leq \iint\left|K_{\tilde{\chi}_{2} R(\lambda, h) \tilde{\chi}_{1}}(x, y) \chi(x, y)\right| d x d y \\
& \leq \iint\left|K_{\rho_{2} R(\lambda, h) \rho_{1}}(x, y)\left(\rho_{2} \otimes \rho_{1}\right)(x, y)\right| d x d y \\
& \leq\left\|\rho_{2}\right\|_{L^{2}\left(\mathbb{R}^{n}\right)}\left\|\rho_{1}\right\|_{L^{2}\left(\mathbb{R}^{n}\right)}\left\|\rho_{2} R(\lambda, h) \rho_{1}\right\|_{\mathcal{B}\left(L^{2}\left(\mathbb{R}^{n}\right)\right)} \\
& =\mathcal{O}\left(h^{s}\right),
\end{aligned}
$$

which verifies the assertion. 
To recall the representation of the resolvent, which we shall use to prove the lemma, we recall that for $f \in C_{c}^{\infty}\left(\mathbb{R}^{n}\right)$

$$
-\frac{i}{h}(P(h)-\lambda) \int_{0}^{T} e^{\frac{i}{h} t \lambda} U(t) f d t=e^{\frac{i}{h} T \lambda} U(T) f-f,
$$

where $U(t)=e^{-\frac{i}{h} t P(h)}, t \in \mathbb{R}$ is the unitary group of $P(h)$. The same proof as in Lemma B.1, [10], shows that $\left(1-\chi_{0}\right) U(T) f \in \mathcal{S}\left(\mathbb{R}^{n}\right), \chi_{0} \in C_{c}^{\infty}\left(\mathbb{R}^{n}\right), \chi_{0}=1$ on $B\left(0, R_{0}\right)$. Since we can also think of $R(\lambda, h)$ as the limit $\lim _{\epsilon \rightarrow 0, \epsilon>0} R(\lambda \pm i \epsilon, h)$ in the spaces of bounded operators $\mathcal{B}\left(L_{\alpha}^{2}, L_{-\alpha}^{2}\right), \alpha>\frac{1}{2}$, where

$$
L_{\alpha}^{2}=\left\{f:\left(\chi_{0}+\left(1-\chi_{0}\right)\langle x\rangle^{\alpha}\right) f \in L^{2}\left(X, d \operatorname{vol}_{g} \mathbb{R}^{n}\right)\right\},
$$

we obtain

$$
\tilde{\chi}_{2} R(\lambda, h) \tilde{\chi}_{1}=\frac{i}{h} \int_{0}^{T} e^{\frac{i}{h} t \lambda} \tilde{\chi}_{2} U(t) \tilde{\chi}_{1} d t+e^{\frac{i}{h} T \lambda} \tilde{\chi}_{2} R(\lambda, h) U(T) \tilde{\chi}_{1}
$$

and this is the representation of the resolvent, which we shall use in this proof.

We further recall the well-known fact that $U(t) \in \mathcal{I}_{h}^{0}\left(\mathbb{R}^{2 n}, \Lambda_{t}\right), t \in \mathbb{R}$, where $\Lambda_{t}=$ graph $\exp \left(t H_{p}\right)$. Since $\rho_{0} \in \Lambda_{R}(\lambda)$ is such that $\gamma\left(\cdot ; \pi_{1}\left(\rho_{0}\right)\right)$ is a non-trapped trajectory, there exists an open set $V \subset \Lambda_{R}(\lambda), \rho_{0} \in V$, such that for every $\rho \in V, \gamma\left(\cdot ; \pi_{1}(\rho)\right)$ is a non-trapped trajectory. By adjusting $V$, if necessary, we can assume that the same holds for all points in $\bar{V}$. Let $Q \in \Psi_{h}^{0}\left(1, \mathbb{R}^{2 n}\right)$ be a microlocal cut-off to the neighborhood $V$ as in (6) with a compactly supported symbol. First, we shall prove that $Q K_{\tilde{\chi}_{2} R(\lambda, h) U(T) \tilde{\chi}_{1}}=\mathcal{O}_{L^{2}\left(\mathbb{R}^{2 n}\right)}\left(h^{\infty}\right)$ for $T>0$ sufficiently large. By [1, Lemma 3, (a)] and the choice of $Q$, we have that

$$
W F_{h}^{i}\left(Q K_{\tilde{\chi}_{2} R(\lambda, h) U(T) \tilde{\chi}_{1}}\right)=\emptyset
$$

and therefore by Proposition 7.1, (i), 9], it follows that it is sufficient to prove that there exists $\bar{T}>0$ such that for every $T>\bar{T}, W F_{h}^{f}\left(Q K_{\tilde{\chi}_{2} R(\lambda, h) e^{-\frac{i}{h} T P(h)} \tilde{\chi}_{1}}\right)=\emptyset$. This will follow, if we prove that $W F_{h}^{f}\left(K_{\tilde{\chi}_{2} R(\lambda, h) e^{-\frac{i}{h} T P(h)} \tilde{\chi}_{1}}\right) \cap W F_{h}^{f}(Q)=\emptyset$. To prove the latter, consider

$$
\left\langle K_{\tilde{\chi}_{2} R(\lambda, h) e^{-\frac{i}{h} T P(h)} \tilde{\chi}_{1}},\left(\psi_{2} \otimes \psi_{1}\right) e^{-\frac{i}{h}(\langle\cdot, \xi\rangle+\langle\cdot, \eta\rangle)}\right\rangle,
$$

where $\operatorname{supp} \psi_{2} \times\{\xi\} \times \operatorname{supp} \psi_{1} \times\{\eta\} \subset V$. Let $V_{1} \subset \mathbb{R}^{n}$ be a bounded open set such that $\operatorname{supp} \psi_{2} \times\{\xi\} \times \operatorname{supp} \psi_{1} \times V_{1} \subset V, \eta \in V_{1}$. Now for every $\eta$ we have that $W F_{h}\left(\psi_{1} e^{-\frac{i}{h}\langle\cdot, \eta\rangle}\right)=$ $\operatorname{supp} \psi_{1} \times\{\eta\}$ is compact. This, together with [1, Theorem 1], 1, Lemma 6], and [1, Lemma 3 , (c)], and the fact that $U(t) \in \mathcal{I}_{h}^{0}\left(\mathbb{R}^{2 n}, \Lambda_{t}\right), t \in \mathbb{R}$, allows us to conclude that

$$
W F_{h}^{f}\left(U(T) \tilde{\chi}_{1} \psi_{1} e^{-\frac{i}{h}\langle\cdot, \eta\rangle}\right) \subset \exp \left(T H_{p}\right)\left(W F_{h}^{f}\left(\psi_{1} e^{-\frac{i}{h}\langle\cdot, \eta\rangle}\right)\right), \eta \in V_{1} .
$$

After decreasing $V_{1}$, if necessary, we have, by the proof of [1, Lemma 4], that the estimates in (33) can be made uniform in $\eta \in V_{1}$. Since $\exp \left(T H_{p}\right)$ is a diffeomorphism, it follows 
that $\cup_{\eta \in \bar{V}_{1}} \exp \left(T H_{p}\right)\left(W F_{h}^{f}\left(\psi_{1} e^{-\frac{i}{h}\langle\cdot, \eta\rangle}\right)\right)$ is compact. Further, as we are working with the outgoing resolvent, we have that

$$
\begin{aligned}
W F_{h}^{f}\left(R(\lambda, h) U(T) \tilde{\chi}_{1} \psi_{1} e^{-\frac{i}{h}\langle\cdot, \eta\rangle}\right) & \subset \cup_{t>0} \exp \left(t H_{p}\right)\left(W F_{h}^{f}\left(U(T) \tilde{\chi}_{1} \psi_{1} e^{-\frac{i}{h}\langle\cdot, \eta\rangle}\right)\right) \\
& \subset \cup_{t>0} \exp \left((t+T) H_{p}\right)\left(W F_{h}^{f}\left(\psi_{1} e^{-\frac{i}{h}\langle\cdot, \eta\rangle}\right)\right) .
\end{aligned}
$$

By the non-trapping assumption, there exists $\bar{T}>0$ such that for every $T>\bar{T}$ and every $(y, \eta) \in \operatorname{supp} \psi_{1} \times V_{1}$ we have $x(T ; y, \eta) \in\left(\operatorname{supp} \tilde{\chi}_{2}\right)^{c}$. We now let $T>\bar{T}$ be fixed and we have

$$
\left\langle K_{\tilde{\chi}_{2} R(\lambda, h) e^{-\frac{i}{h} T P(h)} \tilde{\chi}_{1}},\left(\psi_{2} \otimes \psi_{1}\right) e^{-\frac{i}{h}(\langle\cdot, \xi\rangle+\langle\cdot \cdot, \eta\rangle)}\right\rangle=\mathcal{O}\left(h^{\infty}\right)
$$

for every $\eta \in V_{1}$ and uniformly in $\xi \in U$, where $U \subset \mathbb{R}^{n}$ a bounded open set such that $\operatorname{supp} \psi_{2} \times U \times \operatorname{supp} \psi_{1} \times V_{1} \subset V$. The proof of [1, Lemma 4], now shows again that the estimate here can be made uniform in $\eta \in V_{1}$. We thus have that for every $T>\bar{T}$

$$
W F_{h}^{f}\left(Q K_{\tilde{\chi}_{2} R(\lambda, h) U(T) \tilde{\chi}_{1}}\right)=\emptyset,
$$

which, together with (32), gives

$$
Q K_{\tilde{\chi}_{2} R(\lambda, h) U(T) \tilde{\chi}_{1}}=\mathcal{O}_{L^{2}\left(\mathbb{R}^{2 n}\right)}\left(h^{\infty}\right) .
$$

Let, now, $\tilde{Q} \in \Psi_{h}^{0}\left(1, \mathbb{R}^{2 n}\right)$ have a compactly supported symbol. Then, since $U(t) \in$ $\mathcal{I}_{h}^{0}\left(\mathbb{R}^{2 n}, \Lambda_{t}\right), t \in \mathbb{R}$, we have

$$
\tilde{Q} Q K_{\tilde{\chi}_{2} R(\lambda, h) \tilde{\chi}_{1}}=\frac{i}{h} \int_{0}^{T} e^{\frac{i}{h} t \lambda}\left(\tilde{\chi}_{2} \otimes \tilde{\chi}_{1}\right) K_{U(t)} d t+e^{\frac{i}{h} T \lambda} \tilde{Q} Q K_{\tilde{\chi}_{2} R(\lambda, h) U(T) \tilde{\chi}_{1}}=\mathcal{O}_{L^{2}\left(\mathbb{R}^{2 n}\right)}\left(h^{-1-\frac{n}{2}}\right) \text {. }
$$

Let, now, $B_{j} \in \Psi_{h}^{0}\left(1, \mathbb{R}^{2 n}\right), j=1, \ldots, k$ have compactly supported symbols and principal symbols $b_{j}$ vanishing on $\Lambda_{R}(\lambda)$ and consider

$$
\left(\prod_{j=1}^{k} B_{j}\right) Q K_{\tilde{\chi}_{2} R(\lambda, h) \tilde{\chi}_{1}}
$$

We have that $\Lambda_{R}(\lambda)=\left(\cup_{s \in \mathbb{R}} \Lambda_{s}\right) \cap\left(\Sigma_{\lambda} \times \Sigma_{\lambda}\right)$, and the intersection at every point is clean. Therefore, by Proposition C.3.1 [6], vol. 3, we can choose local coordinates around $\rho$ such that $\cup_{s \in \mathbb{R}} \Lambda_{s}$ and $\Sigma_{\lambda} \times \Sigma_{\lambda}$ are given there by linear equations in the local coordinates. This implies that for every $j=1, \ldots, k$ we can find functions $c_{j}, g_{j}, h_{j} \in C_{c}^{\infty}\left(T^{*} \mathbb{R}^{n}\right)$ with $c_{j}$ vanishing on $\cup_{s \in \mathbb{R}} \Lambda_{s}, j=1, \ldots, k$, such that $b_{j}=c_{j}+((p-\lambda) \otimes 1) g_{j}+(1 \otimes(p-\lambda)) h_{j}$. Now, for $a, b \in S_{2 n}^{0}(1)$ we have that $O p_{h}(a) O p_{h}(b)=O p_{h}(a b)+\mathcal{O}_{\mathcal{B}\left(L^{2}\left(\mathbb{R}^{n}\right)\right)}(h)$ and we can therefore rewrite (36) as follows

$$
\begin{aligned}
\left(\prod_{j=1}^{k} B_{j}\right) Q K_{\tilde{\chi}_{2} R(\lambda, h) \tilde{\chi}_{1}} & \left(\prod _ { j = 1 } ^ { k } \left(O p_{h}\left(c_{j}\right)+O p_{h}\left(g_{j}\right)((P(h)-\lambda) \otimes I)\right.\right. \\
& \left.\left.+O p_{h}\left(h_{j}\right)(I \otimes(P(h)-\lambda))+h S_{j}\right)\right) Q K_{\tilde{\chi}_{2} R(\lambda, h) \tilde{\chi}_{1}}
\end{aligned}
$$


where $S_{j} \in \Psi_{h}^{0}\left(1, \mathbb{R}^{2 n}\right)$ and $\sigma\left(S_{j}\right) \in C_{c}^{\infty}\left(\mathbb{R}^{4 n}\right), j=1, \ldots, k$. This we further rewrite as

$$
\left(\prod_{j=1}^{k} B_{j}\right) Q K_{\tilde{\chi}_{2} R(\lambda, h) \tilde{\chi}_{1}}=\sum_{l=1}^{4^{k}}\left(\prod_{j=1}^{k} T_{j}^{l}\right) Q K_{\tilde{\chi}_{2} R(\lambda, h) \tilde{\chi}_{1}},
$$

where

$$
T_{j}^{l} \in\left\{O p_{h}\left(c_{j}\right), O p_{h}\left(g_{j}\right)((P(h)-\lambda) \otimes I), O p_{h}\left(h_{j}\right)(I \otimes(P(h)-\lambda)), h S_{j}\right\},
$$

$l=1, \ldots, 4^{k}, j=1, \ldots, k$. We now turn to analyzing the individual summands. As the superscript will not be important, we will omit it from the notation.

We consider the case $k>1$. The case $k=1$ will be implicit in the discussion below. Let $m \in\{2, \ldots, k\}$ be the largest index such that

$$
T_{m} \in\left\{O p_{h}\left(c_{m}\right), h S_{m}\right\}
$$

and

$$
T_{m-1} \in\left\{O p_{h}\left(g_{m-1}\right)((P(h)-\lambda) \otimes I), O p_{h}\left(h_{m-1}\right)(I \otimes(P(h)-\lambda))\right\} .
$$

We first assume that $T_{m}=O p_{h}\left(c_{m}\right)$. Since

$$
\sigma_{0}\left(\left[T_{m}, T_{m-1}\right]\right)=\frac{h}{i}\left\{\sigma_{0}\left(T_{m}\right), \sigma_{0}\left(T_{m-1}\right)\right\}
$$

and $\sigma_{0}\left(T_{m}\right)$ and $\sigma_{0}\left(T_{m-1}\right)$ vanish on the Lagrangian submanifold $\Lambda_{R}(\lambda)$ near $\rho$, it follows that $\sigma_{0}\left(\left[T_{m}, T_{m-1}\right]\right)$ also vanishes on $\Lambda_{R}(\lambda)$ near $\rho$. Therefore we have, as before, that

$$
\sigma_{0}\left(\left[T_{m}, T_{m-1}\right]\right)=\tilde{c}+\tilde{g}((p-\lambda) \otimes 1)+\tilde{h}(1 \otimes(p-\lambda))
$$

for some $\tilde{c}, \tilde{g}, \tilde{h} \in C_{c}^{\infty}\left(\mathbb{R}^{4 n}\right)$ with supports in a sufficiently small neighborhood of $\rho$ and $\left.\tilde{c}\right|_{\cup_{s \in \mathbb{R}} \Lambda_{s}}=0$. Thus

$$
\begin{aligned}
T_{m-1} & T_{m} \\
= & O p_{h}(\tilde{c})+O p_{h}(\tilde{g})((P(h)-\lambda) \otimes I)+O p_{h}(\tilde{h})(I \otimes(P(h)-\lambda)) \\
& +T_{m} T_{m-1}+h \tilde{T}
\end{aligned}
$$

where $\tilde{T} \in \Psi_{h}^{0}\left(1, \mathbb{R}^{2 n}\right)$ and $\sigma(\tilde{T}) \in C_{c}^{\infty}\left(\mathbb{R}^{4 n}\right)$ and we use the latter expression in (38) to replace $T_{m-1} T_{m}$ in the product above.

If, now, $T_{m}=h S_{m}$ we rewrite $T_{m-1} T_{m}=T_{m} T_{m-1}+\left[T_{m-1}, T_{m}\right]$ in the product above and observe that $\left[T_{m-1}, T_{m}\right] \in \Psi_{h}^{-2}\left(1, \mathbb{R}^{4 n}\right)$.

We iterate this process until each product which appears in (37), where we may now have more than $4^{k}$ products, is of the form

$$
h^{k-k_{1}} \prod_{j=1}^{k_{1}} Q_{j}, k_{1} \in\{1, \ldots, k\}
$$


where for some $j_{0} \in\left\{0, \ldots, k_{1}\right\}$ we have that

$$
\begin{aligned}
& \text { for } j_{0}<j \leq k_{1}, Q_{j} \in\left\{O p_{h}\left(g_{j}^{1}\right)((P(h)-\lambda) \otimes I), O p_{h}\left(h_{j}^{1}\right)(I \otimes(P(h)-\lambda))\right\}, \\
& \quad \text { for some } g_{j}^{1}, h_{j}^{1} \in C_{c}^{\infty}\left(\mathbb{R}^{4 n}\right), \\
& \text { for } 1 \leq j \leq j_{0}, Q_{j} \in\left\{O p_{h}\left(c_{j}^{1}\right), h S_{j}^{1}\right\}, \\
& \quad \text { for some } c_{j}^{1} \in C_{c}^{\infty}\left(T^{*} \mathbb{R}^{n}\right),\left.c_{j}^{1}\right|_{\cup_{t \in \mathbb{R}} \Lambda_{t}}=0, S_{j}^{1} \in \Psi_{h}^{0}\left(1, \mathbb{R}^{2 n}\right), \sigma\left(S_{j}^{1}\right) \in C_{c}^{\infty}\left(\mathbb{R}^{4 n}\right),
\end{aligned}
$$

with all symbols supported in a sufficiently small neighborhood of $\rho$.

Next, we let $\tilde{m} \in\left\{2, \ldots, k_{1}\right\}$ denote the largest index for which we have $T_{\tilde{m}-1}=$ $O p_{h}\left(c_{\tilde{m}-1}^{1}\right)$ and $T_{\tilde{m}}=h S_{\tilde{m}}^{1}$. We then replace $T_{\tilde{m}-1} T_{\tilde{m}}$ by $T_{\tilde{m}} T_{\tilde{m}-1}+\left[T_{\tilde{m}-1}, T_{\tilde{m}}\right]$ in the product above and observe that $\left[T_{\tilde{m}-1}, T_{\tilde{m}}\right] \in \Psi_{h}^{-1}\left(1, \mathbb{R}^{2 n}\right)$ and $\sigma\left(\left[T_{\tilde{m}-1}, T_{\tilde{m}}\right]\right) \in C_{c}^{\infty}\left(\mathbb{R}^{4 n}\right)$.

We repeat this procedure until every product which appears in (37) is of the form

$$
h^{k-k_{2}} \prod_{j=1}^{k_{2}} V_{j}, k_{2} \in\{1, \ldots, k\},
$$

where for some $j_{1}, j_{2} \in\left\{0, \ldots, k_{2}\right\}, j_{1} \leq j_{2}$, we have that

$$
\begin{aligned}
& \text { for } j_{2}<j \leq k_{2}, V_{j} \in\left\{O p_{h}\left(g_{j}^{2}\right)((P(h)-\lambda) \otimes I), O p_{h}\left(h_{j}^{2}\right)(I \otimes(P(h)-\lambda))\right\}, \\
& \quad \text { for some } g_{j}^{2}, h_{j}^{2} \in C_{c}^{\infty}\left(\mathbb{R}^{4 n}\right), \\
& \text { for } j_{1}<j \leq j_{2}, V_{j}=O p_{h}\left(c_{j}^{2}\right) \text {, for some } c_{j}^{2} \in C_{c}^{\infty}\left(T^{*} \mathbb{R}^{n}\right),\left.c_{j}^{2}\right|_{\cup_{t \in \mathbb{R}} \Lambda_{t}}=0, \\
& \text { for } 1 \leq j \leq j_{1}, V_{j}=h S_{j}^{2}, \text { for some } S_{j}^{2} \in \Psi_{h}^{0}\left(1, \mathbb{R}^{2 n}\right), \sigma\left(S_{j}^{2}\right) \in C_{c}^{\infty}\left(\mathbb{R}^{4 n}\right),
\end{aligned}
$$

where again all symbols are supported in a sufficiently small neighborhood of $\rho$.

We shall again omit the superscripts from the notation below. We also observe that the symmetry of $K_{\tilde{\chi}_{2} R(\lambda, h) \tilde{\chi}_{1}}$ allows us to assume that

$$
V_{j}=O p_{h}\left(g_{j}\right)((P(h)-\lambda) \otimes I), g_{j} \in C_{c}^{\infty}\left(\mathbb{R}^{4 n}\right), j_{2}<j \leq k_{2} .
$$

We now analyze

$$
\begin{aligned}
((P(h)-\lambda) & \otimes I) Q K_{\tilde{\chi}_{2} R(\lambda, h) \tilde{\chi}_{1}} \\
& =[(P(h)-\lambda) \otimes I, Q] K_{\tilde{\chi}_{2} R(\lambda, h) \tilde{\chi}_{1}}+Q\left(\left(-h^{2} \Delta-\lambda\right) \otimes I\right) K_{\tilde{\chi}_{2} R(\lambda, h) \tilde{\chi}_{1}}
\end{aligned}
$$

To analyze the second term, we consider

$$
\begin{aligned}
& \left(-h^{2} \Delta-\lambda\right) \tilde{\chi}_{2} R(\lambda, h) \tilde{\chi}_{1} \\
& \quad=\left(-h^{2} \Delta \tilde{\chi}_{2}\right) R(\lambda, h) \tilde{\chi}_{1}-\left\langle h \nabla \tilde{\chi}_{2}, h \nabla\right\rangle R(\lambda, h) \tilde{\chi}_{1}-\tilde{\chi}_{2}\left(h^{2} \Delta-\lambda\right) R(\lambda, h) \tilde{\chi}_{1} \\
& \quad=\left(-h^{2} \Delta \tilde{\chi}_{2}\right) R(\lambda, h) \tilde{\chi}_{1}-\left\langle h \nabla \tilde{\chi}_{2}, h \nabla\right\rangle R(\lambda, h) \tilde{\chi}_{1}
\end{aligned}
$$

and therefore

$$
\begin{aligned}
& \left\|Q\left(\left(-h^{2} \Delta-\lambda\right) \otimes I\right) K_{\tilde{\chi}_{2} R(\lambda, h) \tilde{\chi}_{1}}\right\|_{L^{2}\left(\mathbb{R}^{2 n}\right)} \\
& \quad \leq C h\left(h\left\|Q K_{\left(\Delta \tilde{\chi}_{2}\right) R(\lambda, h) \tilde{\chi}_{1}}\right\|_{L^{2}\left(\mathbb{R}^{2 n}\right)}+\left\|Q K_{\left\langle\nabla \tilde{\chi}_{2}, h \nabla\right\rangle R(\lambda, h) \tilde{\chi}_{1}}\right\|_{L^{2}\left(\mathbb{R}^{2 n}\right)}\right)
\end{aligned}
$$


Now,

$$
\begin{aligned}
Q K_{\left\langle\nabla \tilde{\chi}_{2}, h \nabla\right\rangle R(\lambda, h) \tilde{\chi}_{1}} & \equiv \frac{i}{h} \int_{0}^{T} e^{\frac{i}{h} t \lambda} Q K_{\left\langle\nabla \tilde{\chi}_{2}, h \nabla\right\rangle U(t) \tilde{\chi}_{1}} d t \\
& =\frac{i}{h} \int_{0}^{T} e^{\frac{i}{h} t \lambda} Q\left(\left\langle\nabla \tilde{\chi}_{2}, h \nabla\right\rangle \otimes \tilde{\chi}_{1}\right) K_{U(t)} d t \\
& =\frac{i}{h} \int_{0}^{T} e^{\frac{i}{h} t \lambda}\left(O p_{h}\left(q\left(\left\langle\nabla \tilde{\chi}_{2}, \xi\right\rangle \otimes \tilde{\chi}_{1}\right)\right)+h O p_{h}(\bar{q})\right) K_{U(t)} d t
\end{aligned}
$$

where the first equality follows from (34) and $\bar{q} \in C_{c}^{\infty}\left(\mathbb{R}^{4 n}\right)$. From

$$
q\left(\left\langle\nabla \tilde{\chi}_{2}, \xi\right\rangle \otimes \tilde{\chi}_{1}\right) \in S_{4 n}^{0}(1) \cap C_{c}^{\infty}\left(\mathbb{R}^{4 n}\right)
$$

it follows that $O p_{h}\left(q\left(\left\langle\nabla \tilde{\chi}_{2}, \xi\right\rangle \otimes \tilde{\chi}_{1}\right)\right)=\mathcal{O}_{\mathcal{B}\left(L^{2}\left(\mathbb{R}^{2 n}\right)\right)}(1)$. Since $U(t) \in \mathcal{I}_{h}^{0}\left(\mathbb{R}^{2 n}, \Lambda_{t}\right), t \in \mathbb{R}$, we then have that

$$
\left\|O p_{h}\left(q\left(\tilde{\chi}_{2} \otimes \tilde{\chi}_{1}\right)(\tilde{p} \otimes 1)\right) K_{U(t)}\right\|_{L^{2}\left(\mathbb{R}^{2 n}\right)}=\mathcal{O}\left(h^{-\frac{n}{2}}\right)
$$

with the norm depending on $t$ continuously. Therefore, from (41), we obtain

$$
\left\|Q K_{\left\langle\nabla \tilde{\chi}_{2}, h \nabla\right\rangle R(\lambda, h) \tilde{\chi}_{1}}\right\|_{L^{2}\left(\mathbb{R}^{2 n}\right)}=\mathcal{O}\left(h^{-\frac{n}{2}-1}\right) .
$$

From

$$
Q K_{\left(\Delta \tilde{\chi}_{2}\right) R(\lambda, h) \tilde{\chi}_{1}} \equiv \frac{i}{h} \int_{0}^{T} e^{\frac{i}{h} t \lambda} Q K_{\left(\Delta \tilde{\chi}_{2}\right) U(t) \tilde{\chi}_{1}} d t
$$

we conclude similarly that

$$
\left\|Q K_{\left(\Delta \tilde{\chi}_{2}\right) R(\lambda, h) \tilde{\chi}_{1}}\right\|_{L^{2}\left(\mathbb{R}^{2 n}\right)}=\mathcal{O}\left(h^{-\frac{n}{2}-1}\right) .
$$

Also in the same way we obtain

$$
[(P(h)-I) \otimes I, Q] K_{\left(\Delta \tilde{\chi}_{2}\right) R(\lambda, h) \tilde{\chi}_{1}}=\mathcal{O}_{L^{2}\left(\mathbb{R}^{2 n}\right)}\left(h^{-\frac{n}{2}}\right)
$$

From (39), (40), (42), (43)), (44), and the fact that $O p_{h}(g)=\mathcal{O}_{\mathcal{B}\left(L^{2}\left(\mathbb{R}^{2 n}\right)\right)}(1)$ for any $g \in$ $C_{c}^{\infty}\left(\mathbb{R}^{4 n}\right)$, we then obtain that

$$
\left\|O p_{h}(g)((P(h)-\lambda) \otimes I) Q K_{\tilde{\chi}_{2} R(\lambda, h) \tilde{\chi}_{1}}\right\|_{L^{2}\left(\mathbb{R}^{2 n}\right)}=\mathcal{O}\left(h^{-\frac{n}{2}}\right)
$$

Let, now, $f \in C_{c}^{\infty}\left(\mathbb{R}^{4 n}\right)$ also have support near $\rho$. Then

$$
\begin{aligned}
& O p_{h}(f)((P(h)-\lambda) \otimes I) O p_{h}(g)((P(h)-\lambda) \otimes I) Q K_{\tilde{\chi}_{2} R(\lambda, h) \tilde{\chi}_{1}} \\
& \quad=O p_{h}(f) O p_{h}(g)((P(h)-\lambda) \otimes I)^{2} Q K_{\tilde{\chi}_{2} R(\lambda, h) \tilde{\chi}_{1}} \\
& \quad+O p_{h}(f)\left[(P(h)-\lambda) \otimes I, O p_{h}(g)\right]((P(h)-\lambda) \otimes I) Q K_{\tilde{\chi}_{2} R(\lambda, h) \tilde{\chi}_{1}} .
\end{aligned}
$$

From (45) and the fact that $\left[(P(h)-\lambda) \otimes I, O p_{h}(g)\right] \in \Psi_{h}^{-1}\left(1, \mathbb{R}^{2 n}\right)$ we obtain

$$
O p_{h}(f)\left[(P(h)-\lambda) \otimes I, O p_{h}(g)\right]((P(h)-\lambda) \otimes I) Q K_{\tilde{\chi}_{2} R(\lambda, h) \tilde{\chi}_{1}}=\mathcal{O}_{L^{2}\left(\mathbb{R}^{2 n}\right)}\left(h^{1-\frac{n}{2}}\right) .
$$


The same argument as in (45) also implies that

$$
O p_{h}(f) O p_{h}(g)((P(h)-\lambda) \otimes I)^{2} Q K_{\tilde{\chi}_{2} R(\lambda, h) \tilde{\chi}_{1}}=\mathcal{O}_{L^{2}\left(\mathbb{R}^{2 n}\right)}\left(h^{1-\frac{n}{2}}\right)
$$

and we obtain, from (46) and (47),

$$
\left\|O p_{h}(f)((P(h)-\lambda) \otimes I) O p_{h}(g)((P(h)-\lambda) \otimes I) Q K_{\tilde{\chi}_{2} R(\lambda, h) \tilde{\chi}_{1}}\right\|_{L^{2}\left(\mathbb{R}^{2 n}\right)}=\mathcal{O}\left(h^{1-\frac{n}{2}}\right) .
$$

Iterating this argument, we then have that

$$
\left\|\left(\prod_{j=j_{2}+1}^{k_{2}} V_{j}\right) Q K_{\tilde{\chi}_{2} R(\lambda, h) \tilde{\chi}_{1}}\right\|_{L^{2}\left(\mathbb{R}^{2 n}\right)}=\mathcal{O}\left(h^{k-j_{2}-1-\frac{n}{2}}\right), h \rightarrow 0 .
$$

We now observe that

$$
\begin{aligned}
\left(\prod_{j=j_{2}+1}^{k_{2}} V_{j}\right) Q K_{\tilde{\chi}_{2} R(\lambda, h) \tilde{\chi}_{1}}= & h^{k_{2}-j_{2}} Q K_{\chi_{3}(h) R(\lambda, h) \tilde{\chi}_{1}}+h^{k_{2}-j_{2}} K_{\chi_{4} \tilde{P} R(\lambda, h) \tilde{\chi}_{1}} \\
& +h^{k_{2}-j_{2}} O p_{h}\left(e_{j_{2}}\right) K_{\tilde{\chi}_{2} \tilde{P} R(\lambda, h) \tilde{\chi}_{1}},
\end{aligned}
$$

where $\chi_{3}(h)$ is polynomial in $h$ with smooth coefficients with supports contained in supp $\tilde{\chi}_{2}$, $\chi_{4} \in C_{c}^{\infty}\left(\mathbb{R}^{n}\right)$ is such that $\operatorname{supp} \chi_{4} \subseteq \operatorname{supp} \tilde{\chi}_{2}, \tilde{P} \in \Psi_{h}^{0}\left(1, \mathbb{R}^{2 n}\right)$ with $\sigma(\tilde{P})$ is supported in a sufficiently small neighborhood of $\rho, e_{j_{2}} \in C_{c}^{\infty}\left(\mathbb{R}^{4 n}\right)$. Therefore

$$
\begin{aligned}
\left(\prod_{i=j_{1}+1}^{j_{2}} V_{i}\right)\left(\prod_{j=j_{2}+1}^{k_{2}} V_{j}\right) K_{\tilde{\chi}_{2} R(\lambda, h) \tilde{\chi}_{1}} \\
\equiv h^{k_{2}-j_{2}-1} i \int_{0}^{T} e^{\frac{i}{h} t \lambda}\left(\prod_{i=j_{1}+1}^{j_{2}} V_{i}\right) Q\left(\chi_{3}(h) \otimes \tilde{\chi}_{1}\right) K_{U(t)} d t \\
\quad+h^{k_{2}-j_{2}-1} i \int_{0}^{T} e^{\frac{i}{h} t \lambda}\left(\prod_{i=j_{1}+1}^{j_{2}} V_{i}\right)\left(\chi_{4} \otimes \tilde{\chi}_{1}\right)(\tilde{P} \otimes I) K_{U(t)} d t \\
\quad+h^{k_{2}-j_{2}-1} i \int_{0}^{T} e^{\frac{i}{h} t \lambda}\left(\prod_{i=j_{1}+1}^{j_{2}} V_{i}\right) O p_{h}\left(e_{j_{2}}\right)\left(\tilde{\chi}_{2} \otimes \tilde{\chi}_{1}\right) K_{U(t)} d t \\
=\mathcal{O}_{L^{2}\left(\mathbb{R}^{2 n}\right)}\left(h^{k_{2}-1-j_{1}-\frac{n}{2}}\right),
\end{aligned}
$$

where we have again used (34) and the fact that $U(t) \in \mathcal{I}_{h}^{0}\left(\mathbb{R}^{2 n}, \Lambda_{t}\right), t \in \mathbb{R}$,.

Lastly, from the fact that $V_{j} \in \Psi_{h}^{-1}\left(1, \mathbb{R}^{2 n}\right), 1 \leq j \leq j_{1}$, we have that

$$
\left(\prod_{j=1}^{k} B_{j}\right) Q K_{\tilde{\chi}_{2} R(\lambda, h) \tilde{\chi}_{1}}=h^{k-k_{2}}\left(\prod_{j=1}^{k_{2}} V_{j}\right) Q K_{\tilde{\chi}_{2} R(\lambda, h) \tilde{\chi}_{1}}=\mathcal{O}_{L^{2}\left(\mathbb{R}^{2 n}\right)}\left(h^{k-1-\frac{n}{2}}\right),
$$

which completes the proof of the theorem. 


\section{$5.2 \quad$ Non-Trapping Energy Level}

Theorem 2 Let $\lambda>0$ be a non-trapping energy level for $P$.

Then amplitude $A(\lambda, h) \in \mathcal{I}_{h}^{\frac{3}{2}}\left(\mathbb{S}^{n-1} \times \mathbb{S}^{n-1}, S R(\lambda)\right)$.

Proof: We recall from [21] that $\left\|\tilde{\chi}_{2} R(\lambda, h) \tilde{\chi}_{1}\right\|_{\mathcal{B}\left(L^{2}\left(\mathbb{R}^{n}\right)\right)}=\mathcal{O}\left(\frac{1}{h}\right)$. Then the result follows from Theorem 1 and the main theorem.

\subsubsection{A Simple Inverse Problem}

Following a suggestion of Plamen Stefanov we include a discussion of an inverse problem motivated by Theorem [2] Suppose that $P=-h^{2} \Delta+V$, where $V$ is compactly supported and smooth, that satisfies the general assumptions of this article. Suppose that $\lambda>\max _{x \in \mathbb{R}^{n}} V_{+}(x)$ so that the energy level $\lambda$ is clearly non-trapping. Let $V$ further be such that the metric $(\lambda-V(x)) d x^{2}$ is simple. We have the following

Theorem 3 For $P$ and $\lambda$ as above, the scattering relation, and hence by Theorem 0 the scattering amplitude, determine $V$ uniquely.

Outline of proof: We compare this with the problem of the wave equation with variable wave speed defined as

$$
c(x)=(\lambda-V(x))^{-\frac{1}{2}} .
$$

Then the function $c$ is equal to $1 / \sqrt{\lambda}$ for large $x$. We have a new Hamiltonian $\widetilde{p}-1=$ $c^{-2}(x)|\xi|^{2}-1$. These Hamiltonians have the same integral curves but they are parameterized in different ways. Hence the scattering relation for this Schrödinger equation is that related to the metric $g_{1}(x)=c^{-2}(x) d x^{2}$. It is now implicit in [1] that the scattering relation for the metric $g_{1}$ determines the boundary distance function uniquely. The results of [13] and 12 further imply that the boundary distance function determines uniquely a simple metric conformal to the Euclidean, in particular, it determines $c$ and therefore $V$.

\subsection{Trapping Energy Level}

Here we consider a trapping energy level $\lambda>0$. We make the following assumption

\section{Assumption 3}

$$
\begin{aligned}
& \exists \epsilon>0: \text { the resonances } \lambda_{j} \text { satisfy } \\
& \left|\Im\left(\lambda_{j}\right)\right| \geq C h^{q} \text { for } \Re\left(\lambda_{j}\right) \in[\lambda-\epsilon, \lambda+\epsilon] .
\end{aligned}
$$

We let $V \subset \Lambda_{R}(\lambda)$ be as in Theorem $\mathbb{1}$ Then for every $(x, \xi) \in \pi_{1}(V)$ there exist unique $t_{j}(x, \xi) \in \mathbb{R}$ such that $\exp \left(t_{j}(x, \xi) H_{p}\right)(x, \xi) \in L_{j}(\lambda), j=1,2$. As in Section 3.1 we have that there exists an open set $U \subset \cup_{(x, \xi) \in \pi_{1}(V)} \exp \left(t_{1}(x, \xi) H_{p}\right)(x, \xi)$ such that we can define the scattering relation $S R_{U}(\lambda)$ as in Section 3.1. By decreasing $U$, if necessary, we can further assume that $S R_{\bar{U}}(\lambda)$ is a Lagrangian submanifold of $T^{*} \mathbb{S}^{n-1} \times T^{*} \mathbb{S}^{n-1}$.

Under these assumptions we have the following 
Theorem $4 A(\lambda, h) \in \mathcal{I}_{h}^{\frac{3}{2}}\left(\mathbb{S}^{n-1} \times \mathbb{S}^{n-1}, S R_{\bar{U}}(\lambda)\right)$.

Proof: Let $C \in \Psi_{h}^{0}\left(1, \mathbb{S}^{n-1} \times \mathbb{S}^{n-1}\right)$ have compactly supported symbol which does not vanish in a neighborhood of a point $\rho \in S R_{\bar{U}}(\lambda)$.

We recall the following result contained in the proof of Proposition 5.1, [9].

Proposition 1 Let Assumption 3 hold. Then there exists $\tilde{n} \in \mathbb{N}$ such that

$$
\|\chi R(z, h) \chi\|_{\mathcal{B}\left(L^{2}\left(\mathbb{R}^{n}\right)\right)}=\mathcal{O}\left(h^{-\tilde{n}}\right), h \rightarrow 0,
$$

uniformly for $z \in\left\{z \in \mathbb{C}:(\Re z, \pm \Im z) \in\left(\frac{1}{d}, d\right) \times(0,1]\right\}$ and $\chi \in C_{c}^{\infty}\left(\mathbb{R}^{n}\right), \chi=1$ on $B(0, \rho)$, for $\rho$ large enough.

This Proposition was proved in [9] for a certain class of potential perturbations of the Euclidean Laplacian. The proof there, however, relies only on Lemma 4.1, [19], and the latter is proved in the black-box setting. Therefore the above Proposition holds also in the setting discussed here.

From Theorem 11 we have that $\tilde{\chi}_{2} R(\lambda, h) \tilde{\chi}_{1} \in \mathcal{I}_{h}^{1}\left(\mathbb{R}^{2 n}, \bar{V} \cap \Lambda_{R}(\lambda)\right)$ and the assertion of the Theorem now follows by the main theorem.

\subsection{Microlocal Representation of the Scattering Amplitude}

Here we prove the following

Theorem 5 Let $\omega_{0} \in \mathbb{S}^{n-1}$ be regular for $\theta_{0} \in \mathbb{S}^{n-1}$ and $L \in \mathbb{N}$ be the number of $\left(\theta_{0}, \omega_{0}\right)$ phase trajectories. Let $P_{l} \in \Psi_{h}^{0}\left(1, \mathbb{S}^{n-1} \times \mathbb{S}^{n-1}\right), l=1, \ldots, L$, be microlocal cut-offs to the Lagrangian submanifolds $S R_{l}(\lambda)$ defined by (19), respectively.

Then

$$
P_{l} A(\lambda, h)=e^{\frac{i}{h} S_{l}} a_{l}, l=1, \ldots, L,
$$

where $S_{l}, l=1, \ldots, L$, are as given by (21) and $a_{l} \in S_{2 n-2}^{\frac{n}{2}+1}(1), l=1, \ldots, L$, have compact support.

Proof: By Theorem 2 and Theorem 4 , the scattering amplitude is a global semi-classical Fourier integral operator associated to $\cup_{l=1}^{L} S R_{l}(\lambda)$. The assertion of the Theorem then follows from Lemma 4, [1, Lemma 5], and [1, Theorem 1].

We remark here that the phase function in this microlocal representation of the scattering amplitude is the same as the one given by [9] and [16].

Acknowledgements. I would like to thank Maciej Zworski for supervising my Ph. D. thesis of which this paper formed a part. I would also like to thank Vesselin Petkov for introducing me to the $\mathrm{Ph}$. D. thesis of his student Laurent Michel, which has helped me complete my work on this project and Plamen Stefanov for discussions leading to the material presented in Section 5.2.1. I am further grateful to Victor Ivrii and Xiang Tang for helpful discussions. 


\section{References}

[1] Alexandrova, Ivana. Semi-Classical Wavefront Set and Fourier Integral Operators. Preprint mathAP.0407460 on arxiv.org.

[2] Arnold, Vladimir. Mathematical Methods of Classical Mechanics; Springer-Verlag: New York, 1980.

[3] Burq, Nicolas. Semi-classical Estimates for the Resolvent in Nontrapping Geometries. International Mathematics Research Notices 2002, (5), 221-241.

[4] Dimassi, Mouez; Sjöstrand, Johannes. Spectral Asymptotics in the Semi-Classical Limit; Cambridge University Press: Cambridge, 1999.

[5] Guillemin, Victor. Sojourn Times and Asymptotic Properties of the Scattering Matrix. Publications of the Research Institute for Mathematical Sciences, Supplement 1977, 12, $69-88$.

[6] Hörmander, Lars. The Analysis of Linear Partial Differential Operators; Springer Verlag: Berlin, 1980.

[7] Majda, Andrew. High Frequency Asymptotics for the Scattering Matrix and the Inverse Problem of Acoustical Scattering. Communications on Pure and Applied Mathematics 1976, 29 (3), 261-291.

[8] Melrose, Richard. Geometric Scattering Theory; Cambridge University Press: Cambridge, 1995.

[9] Michel, Laurent. Semi-classical Behavior of the Scattering Amplitude for Trapping Perturbations at Fixed Energy. Canadian Journal of Mathematics. To appear.

[10] Michel, Laurent. Semi-classical Limit of the Scattering Amplitude for Trapping Perturbations. Asymptotic Analysis 2002, 32 (3-4), 221-255.

[11] Michel, René. Sur la rigidité imposée par la longueur des déodésiques. Inventiones Mathematicae 1981, 65 (1), 71-83.

[12] Mukhometov, R. G. A Problem of Reconstructing a Riemannian Metric. Siberian Mathematical Journal 1981, 22 (3), 420-433.

[13] Mukhometov, R. G.; Romanov, V. G. On the problem of finding an isotropic Riemannian metric in an $n$-dimensional space. (Russian) Doklady Akademii Nauk SSSR 1978, 243 (1), 41-44.

[14] Petkov, Vesselin; Zworski, Maciej. Semi-Classical Estimates on the Scattering Determinant. Annales Henri Poincaré 2001, 2 (4), 675-711. 
[15] Protas, Yuri. Quasiclassical Asymptotic Behavior of the Scattering Amplitude of a Plane Wave on the Inhomogeneities of a Medium. Mathematics of the USSR - Sbornik 1982, 117 (159) (4), 494-515 (560).

[16] Robert, Didier; Tamura, Hideo. Asymptotic Behavior of Scattering Amplitudes in SemiClassical and Low Energy Limits. Annales de l'Institut Fourier 1989, 39 (1), 155-192.

[17] Sjöstrand, Johannes; Zworski, Maciej. Complex Scaling and the Distribution of Scattering Poles. Journal of the American Mathematical Society 1991, 4 (4), 729-769.

[18] Stefanov, Plamen. Estimates on the Residue of the Scattering Matrix. Asymptotic Analysis 2002, 32 (3-4), 317-333.

[19] Tang, Siu-Hung; Zworski, Maciej. From Quasimodes to Resonances. Mathematical Research Letters 1998, 5 (3), 261-272.

[20] Vainberg, Boris. Quasiclassical Approximation in Stationary Scattering Problems. Functional Analysis and its Applications 1977, 11 (4), 6-18.

[21] Vasy, András; Zworski, Maciej. Semiclassical Estimates in Asymptotically Euclidean Scattering. Communications in Mathematical Physics 2000, 212 (1), 205-217.

[22] Yajima, Kenji. The Quasiclassical Limit of Scattering Amplitude. $L^{2}$ Approach for Short Range Potentials. Japanese Journal of Mathematics 1987, 13 (1), 77-126. 